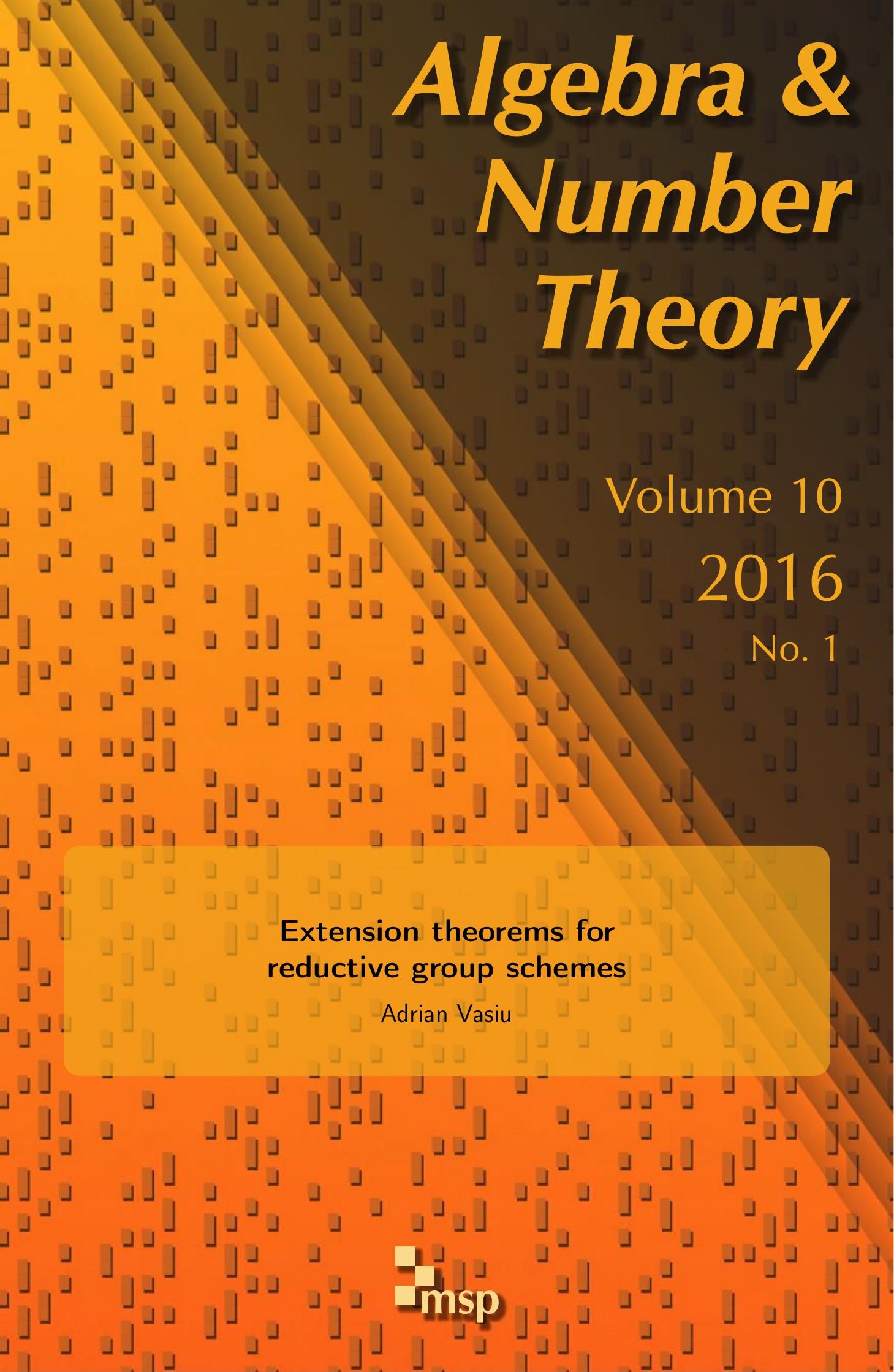




\title{
Extension theorems for reductive group schemes
}

\author{
Adrian Vasiu
}

\begin{abstract}
We prove several basic extension theorems for reductive group schemes via extending Lie algebras and via taking schematic closures. We also prove that, for each scheme $Y$, the category in groupoids of adjoint group schemes over $Y$ whose Lie algebra $\mathrm{O}_{Y}$-modules have perfect Killing forms is isomorphic, via the differential functor, to the category in groupoids of Lie algebra $\mathrm{O}_{Y}$-modules which have perfect Killing forms and which, as $\mathrm{O}_{Y}$-modules, are coherent and locally free.
\end{abstract}

\section{Introduction}

A group scheme $H$ over a scheme $S$ is called reductive if the morphism $H \rightarrow S$ has the following two properties: (i) it is smooth and affine (and therefore of finite presentation), and (ii) its geometric fibers are reductive groups over spectra of fields and therefore are connected (see [SGA $3_{\text {III }}$ 1970, Exposé XIX, Sections 2.7, 2.1, and 2.9]). If, moreover, the center of $H$ is trivial, then $H$ is called an adjoint group scheme over $S$. Let $O_{S}$ be the structure ring sheaf of $S$. Let $\operatorname{Lie}(H)$ be the Lie algebra $\mathrm{O}_{S}$-module of $H$. As an $\mathrm{O}_{S}$-module, $\operatorname{Lie}(H)$ is coherent and locally free.

The main goal of the paper is to prove Theorems 1.2 and 1.4 below (see Sections 3 and 4) and to apply them and their proofs to obtain new extension theorems for homomorphisms between reductive group schemes (see Section 5). We begin by introducing two groupoids on sets (i.e., two categories whose morphisms are all isomorphisms).

1.1. Two groupoids on sets. Let $Y$ be an arbitrary scheme. Let $\operatorname{Adj}^{-p e r f} f_{Y}$ be the category whose objects are adjoint group schemes over $Y$ with the property that their Lie algebra $\mathrm{O}_{Y}$-modules have perfect Killing forms (i.e., the Killing forms induce naturally $\mathrm{O}_{Y}$-linear isomorphisms from them into their duals) and whose morphisms are isomorphisms of group schemes. Let Lie-perf ${ }_{Y}$ be the category whose objects are Lie algebra $\mathrm{O}_{Y}$-modules which have perfect Killing forms and

MSC2010: primary 14L15; secondary 11G18, 14F30, 14G35, 14K10, 14L17, 17B45.

Keywords: reductive group schemes, purity, regular rings, Lie algebras. 
which as $\mathrm{O}_{Y}$-modules are coherent and locally free and whose morphisms are isomorphisms of Lie algebra $\mathrm{O}_{Y}$-modules.

Theorem 1.2. Let $\mathscr{L}_{Y}: \operatorname{Adj}_{-} \operatorname{perf}_{Y} \rightarrow$ Lie-perf $_{Y}$ be the functor which associates to a morphism $f: G \stackrel{\sim}{\longrightarrow} H$ of $\operatorname{Adj-perf~}_{Y}$ the morphism $d f: \operatorname{Lie}(G) \stackrel{\sim}{\longrightarrow} \operatorname{Lie}(H)$ of Lie-perf $f_{Y}$ which is the differential of $f$. Then the functor $\mathscr{L}_{Y}$ is an equivalence of categories.

We have a variant of this theorem for simply connected semisimple group schemes instead of adjoint group schemes; see Corollary 3.7. This theorem implies the classification of Lie algebras over fields of characteristic at least 3 that have nondegenerate Killing forms obtained previously by Curtis [1957], Seligman [1967], Mills [1957], Mills and Seligman [1957], Block-Zassenhaus [1964], and Brown [1969] (see Remark 3.6(a)). The functor $\mathscr{L}_{Y}$ is an equivalence of nonempty categories if and only if $Y$ is a Spec $Z\left[\frac{1}{2}\right]$-scheme; see Corollary 3.8. Directly from Theorem 1.2 we get our first extension result:

Corollary 1.3. We assume that $Y=\operatorname{Spec} A$ is an affine scheme. Let $K$ be the ring of fractions of $A$. Let $G_{K}$ be an adjoint group scheme over Spec $K$ such that the symmetric bilinear Killing form on the Lie algebra $\operatorname{Lie}\left(G_{K}\right)$ of $G_{K}$ is perfect (i.e., it induces naturally a K-linear isomorphism $\left.\operatorname{Lie}\left(G_{K}\right) \stackrel{\sim}{\longrightarrow} \operatorname{Hom}_{K}\left(\operatorname{Lie}\left(G_{K}\right), K\right)\right)$. We assume that there exists a Lie algebra $\mathfrak{g}$ over A such that the following two properties hold:

(i) we have an identity $\operatorname{Lie}\left(G_{K}\right)=\mathfrak{g} \otimes_{A} K$ and the A-module $\mathfrak{g}$ is projective and finitely generated;

(ii) the symmetric bilinear Killing form on $\mathfrak{g}$ is perfect.

Then there exists a unique adjoint group scheme $G$ over $Y$ that extends $G_{K}$, with an identity $\operatorname{Lie}(G)=\mathfrak{g}$ that extends the identity of property (i).

Let $U$ be an open, Zariski-dense subscheme of $Y$. We call the pair $(Y, Y \backslash U)$ quasipure if each finite étale cover of $U$ extends uniquely to a finite étale cover of $Y$ (to be compared with [SGA 2 1968, Exposé X, Definition 3.1]).

Theorem 1.4. We assume that $Y$ is a normal, noetherian scheme and the codimension of $Y \backslash U$ in $Y$ is at least 2. Then the following two properties hold:

(a) Let $G_{U}$ be an adjoint group scheme over $U$. We assume that the Lie algebra $O_{U^{-}}$ module Lie $\left(G_{U}\right)$ of $G_{U}$ extends to a Lie algebra $O_{Y}$-module that is a locally free $O_{Y}$-module. Then $G_{U}$ extends uniquely to an adjoint group scheme $G$ over $Y$.

(b) Let $H_{U}$ be a reductive group scheme over $U$. We assume that the pair $(Y, Y \backslash U)$ is quasipure and that the Lie algebra $\mathrm{O}_{U}$-module Lie $\left(G_{U}\right)$ of the adjoint group scheme $G_{U}$ of $H_{U}$ extends to a Lie algebra $\mathrm{O}_{Y}$-module that is a locally free $\mathrm{O}_{Y}$-module. Then $\mathrm{H}_{U}$ extends uniquely to a reductive group scheme $H$ over $Y$. 
The proof of Theorem 1.2 that we include combines the cohomology theory of Lie algebras with a simplified variant of [Vasiu 1999, Claim 2, p. 464] (see Theorem 3.3 and Section 3.4). The proof of Theorem 1.4(a) is an application of [Colliot-Thélène and Sansuc 1979, Corollary 6.12] (see Section 4.1). The classical purity theorem of Nagata and Zariski (see [SGA 2 1968, Exposé X, Theorem 3.4(i)]) says that the pair $(Y, Y \backslash U)$ is quasipure, provided $Y$ is regular and $U$ contains all points of $Y$ of codimension 1 in $Y$. In such a case, a slightly weaker form of Theorem 1.4(b) was obtained in [Colliot-Thélène and Sansuc 1979, Theorem 6.13]. In general, the hypotheses of Theorem 1.4 are needed (see Remark 4.3). See [Moret-Bailly 1985] (resp. [Faltings and Chai 1990; Vasiu 1999; 2004; Vasiu and Zink 2010]) for different analogues of Theorem 1.4 for Jacobian (resp. abelian) schemes. For instance, in [Vasiu and Zink 2010, Corollary 1.5] it is proved that if $Y$ is a regular, formally smooth scheme over the spectrum of a discrete valuation ring of mixed characteristic $(0, p)$ and index of ramification at most $p-1$ and if $U$ contains all points of $Y$ that are of either characteristic 0 or codimension 1 in $Y$, then each abelian scheme over $U$ extends uniquely to an abelian scheme over $Y$.

Notation and basic results are presented in Section 2. In Section 3 we prove Theorem 1.2. In Section 4 we prove Theorem 1.4.

Section 5 contains two results on extending homomorphisms between reductive group schemes. Proposition 5.1 is an application of Theorem 1.4(b) and pertains to extensions of homomorphisms in codimension at least 2 over normal bases. Proposition 5.2 pertains to extensions of homomorphisms via schematic closures and refines [Vasiu 1999, Lemma 3.1.6]; its role is to achieve natural reductions such as the reduction to the case of either a torus or a semisimple group scheme.

Our main motivation for Theorems 1.2 and 1.4 stems from the meaningful applications to crystalline cohomology one gets by combining them with either Faltings' results [1999, Section 4] (see [Vasiu 1999; 2008]) or de Jong's extension theorem [1998, Theorem 1.1] (see [Vasiu 2012a; 2012b]). The manuscripts [Vasiu 2012a; 2012b] apply the results of the current paper to extend our prior work on integral canonical models of Shimura varieties of Hodge type in unramified mixed characteristic $(0, p)$ with $p \geq 5$ (see [Vasiu 1999]) to unramified mixed characteristics $(0,2)$ and $(0,3)$. In addition, this paper can be used to get relevant simplifications of certain parts of the mentioned prior work (see [Vasiu 2008]).

\section{Preliminaries}

Our notation is gathered in Section 2.1, and then we include four basic results that are often used in Sections 3 to 5.

2.1. Notation and conventions. Let $\bar{K}$ be an algebraic closure of a field $K$. Let $H$ be a reductive group scheme over a scheme $S$. Let $Z(H), H^{\text {der }}, H^{\text {ad }}$, and $H^{\text {ab }}$ 
denote the center, the derived group scheme, the adjoint group scheme, and the abelianization of $H$ (respectively). We have $H^{\mathrm{ab}}=H / H^{\mathrm{der}}$ and $H^{\mathrm{ad}}=H / Z(H)$. The center $Z(H)$ is a group scheme of multiplicative type; see [SGA $3_{\text {III }} 1970$, Exposé XXII, Corollary 4.1.7]. Let $Z^{0}(H)$ be the maximal torus of $Z(H)$; the quotient group scheme $Z(H) / Z^{0}(H)$ is a finite, flat group scheme over $S$ of multiplicative type. Let $H^{\mathrm{sc}}$ be the simply connected semisimple group scheme cover of the derived group scheme $H^{\text {der }}$.

See [SGA $3_{\text {III }}$ 1970, Exposé XXII, Corollary 4.3.2] for the quotient group scheme $H / F$ of $H$ by a flat, closed, multiplicative type subgroup scheme $F$ of $Z(H)$.

If $X$ or $X_{S}$ is an $S$-scheme, let $X_{A_{1}}$ (resp. $X_{S_{1}}$ ) be its pullback via a morphism Spec $A_{1} \rightarrow S$ (resp. $S_{1} \rightarrow S$ ).

If $S$ is either affine or integral, let $K_{S}$ be the ring of fractions of $S$. If $S$ is a normal, noetherian, integral scheme, let $\mathscr{D}(S)$ be the set of local rings of $S$ that are discrete valuation rings.

Let $\mathbb{G}_{m, S}$ be the rank-1 split torus over $S$; similarly, the group schemes $\mathbb{G}_{a, S}$, $\mathrm{GL}_{d, S}$ with $d \in \mathbb{N}^{*}$, etc., will be understood to be over $S$. Let Lie $(H)$ be the Lie algebra $O_{S}$-module of $H$. If $S=\operatorname{Spec} A$ is affine, then let $\mathbb{G}_{m, A}:=\mathbb{G}_{m, S}$, etc., and let $\operatorname{Lie}(F)$ be the Lie algebra over $A$ of a closed subgroup scheme $F$ of $H$. For $A$ modules, we have $\operatorname{Lie}(F)=\operatorname{Ker}\left(F\left(A[x] / x^{2}\right) \rightarrow F(A)\right)$, where the $A$-epimorphism $A[x] /\left(x^{2}\right) \rightarrow A$ takes $x$ to 0 . The Lie bracket on $\operatorname{Lie}(F)$ is defined by taking the (total) differential of the commutator morphism [, ] :F $\times_{S} F \rightarrow F$ at identity sections. If $S=\operatorname{Spec} A$ is affine, then $\operatorname{Lie}(H)=\operatorname{Lie}(H)(S)$ is the Lie algebra over $A$ of global sections of $\operatorname{Lie}(H)$ and it is a projective, finitely generated $A$-module.

If $N$ is a projective, finitely generated $A$-module, let $N^{*}:=\operatorname{Hom}_{A}(N, A)$, let $\mathrm{GL}_{N}$ be the reductive group scheme over Spec $A$ of linear automorphisms of $N$, and let $\mathfrak{g l}_{N}:=\operatorname{Lie}\left(\mathrm{GL}_{N}\right)$. Thus $\mathfrak{g l}_{N}$ is the Lie algebra associated to the $A$-algebra $\operatorname{End}_{A}(N)$. A bilinear form $b_{N}: N \times N \rightarrow A$ on $N$ is called perfect if it induces an $A$-linear map $N \rightarrow N^{*}$ that is an isomorphism. If $b_{N}$ is symmetric, then its kernel is the $A$-submodule

$$
\operatorname{Ker}\left(b_{N}\right):=\left\{a \in N \mid b_{N}(a, b)=0 \text { for all } b \in N\right\}
$$

of $N$. For a Lie algebra $\mathfrak{g}$ over $A$ that is a projective, finitely generated $A$-module, let ad $: \mathfrak{g} \rightarrow \mathfrak{g l}_{\mathfrak{g}}$ be the adjoint representation of $\mathfrak{g}$ and let $\mathcal{K}_{\mathfrak{g}}: \mathfrak{g} \times \mathfrak{g} \rightarrow A$ be the Killing form on $\mathfrak{g}$. For $a, b \in \mathfrak{g}$ we have $\operatorname{ad}(a)(b)=[a, b]$, and $\mathscr{K}_{\mathfrak{g}}(a, b)$ is the trace of the endomorphism $\operatorname{ad}(a) \circ \operatorname{ad}(b)$ of $\mathfrak{g}$. The kernel $\operatorname{Ker}\left(\mathscr{K}_{\mathfrak{g}}\right)$ is an ideal of $\mathfrak{g}$.

We denote by $k$ an arbitrary field. Let $n \in \mathbb{N}^{*}$. See [Bourbaki 2002, Chapter VI, Section 4] and [Humphreys 1972, Chapter III, Section 11] for the classification of connected Dynkin diagrams. For

$$
b \in\left\{A_{n}, B_{n}, C_{n} \mid n \in \mathbb{N}^{*}\right\} \cup\left\{D_{n} \mid n \geq 3\right\} \cup\left\{E_{6}, E_{7}, E_{8}, F_{4}, G_{2}\right\},
$$


we say that $H$ is of isotypic b Dynkin type if the connected Dynkin diagram of each simple factor of an arbitrary geometric fiber of $H^{\text {ad }}$ is b; if $H^{\text {ad }}$ is absolutely simple, we drop the word 'isotypic'. We recall that $A_{1}=B_{1}=C_{1}, B_{2}=C_{2}$, and $A_{3}=D_{3}$.

Proposition 2.2. Let $Y$ be a normal, noetherian, integral scheme. Let $K:=K_{Y}$.

(a) If $Y=\operatorname{Spec} A$ is affine, then inside the field $K$ we have $A=\bigcap_{V \in \mathscr{D}(Y)} V$.

(b) Let $U$ be an open subscheme of $Y$ such that $Y \backslash U$ has codimension in $Y$ at least 2. Let $W$ be an affine $Y$-scheme of finite type. Then the natural restriction map $\operatorname{Hom}_{Y}(Y, W) \rightarrow \operatorname{Hom}_{Y}(U, W)$ is a bijection. If, moreover, $W$ is integral, normal and such that we have $\mathscr{D}(W)=\mathscr{D}\left(W_{U}\right)$, then $W$ is determined (up to unique isomorphism) by $W_{U}$.

(c) Suppose that $Y=\operatorname{Spec} A$ is local, regular, and has dimension 2. Let $y$ be the closed point of $Y$ and let $U:=Y \backslash\{y\}$. Then each locally free $\mathrm{O}_{U}$-module of finite rank extends uniquely to a free $\mathrm{O}_{Y}$-module.

Proof. See [Matsumura 1980, Theorem 38] for (a). To check (b), we can assume $Y=\operatorname{Spec} A$ is affine. We write $W=\operatorname{Spec} B$. The $A$-algebra of global functions of $U$ is $A$; see (a). We have $\operatorname{Hom}_{Y}(U, W)=\operatorname{Hom}_{A}(B, A)=\operatorname{Hom}_{Y}(Y, W)$. If, moreover, $B$ is a normal ring and we have $\mathscr{D}(W)=\mathscr{D}\left(W_{U}\right)$, then $B$ is uniquely determined by $\mathscr{D}\left(W_{U}\right)$ (see (a)) and therefore by $W_{U}$. From this (b) follows. See [SGA 2 1968, Exposé X, Lemma 3.5] for (c).

Proposition 2.3. Let $G$ be a reductive group scheme over a scheme $Y$. Then the functor on the category of $Y$-schemes that parametrizes maximal tori of $G$ is representable by a smooth, separated $Y$-scheme of finite type. Thus $G$ has split, maximal tori, locally in the étale topology of $Y$.

Proof. See [SGA $3_{\text {II }}$ 1970, Exposé XII, Corollary 1.10] for the first part. The second part follows easily from the first part (see also [SGA $3_{\text {III }}$ 1970, Exposé XIX, Proposition 6.1]).

Lemma 2.3.1. Let $Y$ be a reduced scheme. Let $G$ be a reductive group scheme over $Y$. Let $K:=K_{Y}$. Let $f_{K}: G_{K}^{\prime} \rightarrow G_{K}$ be a central isogeny of reductive group schemes over Spec $K$. We assume that either $G$ is split or $Y$ is normal. We have:

(a) There exists (up to a canonical identification) at most one central isogeny $f: G^{\prime} \rightarrow G$ that extends $f_{K}: G_{K}^{\prime} \rightarrow G_{K}$. If $Y$ is integral (i.e., $K$ is a field), then there exists a unique central isogeny $f: G^{\prime} \rightarrow G$ that extends $f_{K}: G_{K}^{\prime} \rightarrow G_{K}$.

(b) If $Y$ is normal and integral, then $G^{\prime}$ is the normalization of $G$ in (the field of fractions of ) $G_{K}^{\prime}$.

Proof. We first prove (a) in the case when $G$ is split. Let $T$ be a split, maximal torus of $G$. We first prove the existence part; thus $K$ is a field. As $f_{K}$ is a central isogeny, the inverse image $T_{K}^{\prime}$ of $T_{K}$ in $G_{K}^{\prime}$ is a split torus. Thus $G_{K}^{\prime}$ is split. Let $\mathscr{R}^{\prime} \rightarrow \mathscr{R}$ be 
the 1-morphism of root data in the sense of [SGA $3_{\text {III }}$ 1970, Exposé XXI, Definition 6.8.1] which is associated to the central isogeny $f_{K}: G_{K}^{\prime} \rightarrow G_{K}$ that extends the isogeny $T_{K}^{\prime} \rightarrow T_{K}$. Let $\tilde{f}: \tilde{G}^{\prime} \rightarrow G$ be a central isogeny of split, reductive group schemes over $Y$ which extends an isogeny of split tori $\tilde{T}^{\prime} \rightarrow T$ and for which the 1-morphism of root data associated to it and to the isogeny $\tilde{T}^{\prime} \rightarrow T$ is $\mathscr{R}^{\prime} \rightarrow \mathscr{R}$ (see [SGA $3_{\text {III }}$ 1970, Exposé XXV, Theorem 1.1]). From loc. cit. we also get that there exists an isomorphism $i_{K}: \tilde{G}_{K}^{\prime} \stackrel{\sim}{\longrightarrow} G_{K}^{\prime}$ such that we have $\tilde{f}_{K}=f_{K} \circ i_{K}$. Obviously, $i_{K}$ is unique. Let $G^{\prime}$ be the unique group scheme over $Y$ such that $i_{K}$ extends (uniquely) to an isomorphism $i: \tilde{G}^{\prime} \stackrel{\sim}{\longrightarrow} G^{\prime}$. Let $f:=\tilde{f} \circ i^{-1}: G^{\prime} \rightarrow G$ be a central isogeny.

To check the uniqueness part, we consider two central isogenies $G^{\prime} \rightarrow G$ and $G_{1}^{\prime} \rightarrow G$ that extend a central isogeny $f_{K}: G_{K}^{\prime} \rightarrow G_{K}$ (thus $G_{K}^{\prime}=G_{1, K}^{\prime}$ ). Let $G_{2}^{\prime}$ be the schematic closure of $G_{K}^{\prime}$ embedded diagonally into the product $G^{\prime} \times{ }_{Y} G_{1}^{\prime}$. We are left to check that the two projections $\pi_{1}: G_{2}^{\prime} \rightarrow G^{\prime}$ and $\pi_{2}: G_{2}^{\prime} \rightarrow G_{1}^{\prime}$ are isomorphisms, as in such a case the composite isomorphism $\pi_{2} \circ \pi_{1}^{-1}: G^{\prime} \stackrel{\sim}{\longrightarrow} G_{1}^{\prime}$ is an isomorphism that extends the identity $G_{K}^{\prime}=G_{1, K}^{\prime}$. This statement is local for the étale topology of $Y$, and therefore we can assume, based on Proposition 2.3, that the inverse images of $T$ to $G^{\prime}$ and $G_{1}^{\prime}$ are split tori. From this and [SGA $3_{\text {III }}$ 1970, Exposé XXIII, Theorem 4.1] we get that there exists a unique isomorphism $\theta: G_{1} \stackrel{\sim}{\rightarrow} G_{1}^{\prime}$ which extends the identity $G_{K}^{\prime}=G_{K}$. This implies that $G_{2}^{\prime}$ is the graph of $\theta$, and therefore the two projections $\pi_{1}$ and $\pi_{2}$ are isomorphisms. We conclude that (a) holds if $G$ is split.

We now prove simultaneously (a) and (b) in the case when $Y$ is normal. If a $G^{\prime}$ as in (a) exists, then it is a smooth scheme over the normal scheme $Y$ and thus it is a normal scheme; from this and the fact that $f: G^{\prime} \rightarrow G$ is a finite morphism, we get that $G^{\prime}$ is the normalization of $G$ in $G_{K}^{\prime}$ and, in particular, that it is unique.

Thus to finish the proof of the lemma, it suffices to show that the normalization $G^{\prime}$ of $G$ in $G_{K}^{\prime}$ is a reductive group scheme equipped with a central isogeny $f: G^{\prime} \rightarrow G$, locally in the étale topology of $Y$. As each connected, étale scheme over $Y$ is a normal, integral scheme, based on Proposition 2.3 we can assume that $G$ has a split, maximal torus $T$. Thus the fact that $G^{\prime}$ is a reductive group scheme equipped with a central isogeny $f: G^{\prime} \rightarrow G$ follows from the previous three paragraphs.

Lemma 2.3.2. Let $Y=\operatorname{Spec} A$ be an affine scheme. Let $K:=K_{Y}$. Let $T$ be a torus over $Y$ equipped with a homomorphism $\rho: T \rightarrow G$, where $G$ is a reductive group scheme over $Y$. Then the following three properties hold:

(a) the kernel $\operatorname{Ker}(\rho)$ is a group scheme over $Y$ of multiplicative type;

(b) the kernel $\operatorname{Ker}(\rho)$ is trivial (resp. finite) if and only if the kernel $\operatorname{Ker}\left(\rho_{K}\right)$ is trivial (resp. finite);

(c) the quotient group scheme $T / \operatorname{Ker}(\rho)$ is a torus and we have a closed embedding homomorphism $T / \operatorname{Ker}(\rho) \hookrightarrow G$. 
Proof. The statements of the lemma are local for the étale topology of $Y$. Thus we can assume that $Y$ is local and (see Proposition 2.3) that $T$ and $G$ are split. As $Y$ is connected, the split reductive group scheme $G$ has constant Lie type. Thus $G$ is the pullback to $Y$ of a reductive group scheme $G_{\mathbb{Z}}$ over Spec $\mathbb{Z}$; see [SGA $3_{\text {III }}$ 1970, Exposé XXV, Corollary 1.2]. As $G_{\mathbb{Z}}$ can be embedded into a general linear group scheme over Spec $\mathbb{Z}$ (for instance, see [SGA 3 I 1970, Exposé VI B $_{\mathrm{B}}$, Remark 11.11.1]), there exists a closed embedding homomorphism $G \hookrightarrow \mathrm{GL}_{M}$, with $M$ a free $A$-module of rank $d \in \mathbb{N}^{*}$. By replacing $\rho$ with its composite with this closed embedding homomorphism $G \hookrightarrow \mathrm{GL}_{M}$, we can assume that $G=\mathrm{GL}_{M}$ is a general linear group scheme over $Y$. The representation of $T$ on $M$ is a finite direct sum of representations of $T$ of rank 1; see [Jantzen 2003, Part I, Section 2.11]. Thus $\rho$ factors as the composite of a homomorphism $\rho_{1}: T \rightarrow \mathbb{G}_{m, A}^{m}$ with a closed embedding homomorphism $\mathbb{G}_{d, A}^{m} \hookrightarrow \mathrm{GL}_{M}$. The kernel $\operatorname{Ker}\left(\rho_{1}\right)$ is a group scheme over $Y$ of multiplicative type; see [SGA $3_{\text {II }}$ 1970, Exposé IX, Proposition 2.7(i)]. As $\operatorname{Ker}(\rho)=\operatorname{Ker}\left(\rho_{1}\right)$, we get that (a) holds. As (a) holds, $\operatorname{Ker}(\rho)$ is flat over $Y$ as well as the extension of a finite, flat group scheme $T_{1}$ by a torus $T_{0}$. But $T_{1}$ (resp. $T_{0}$ ) is a trivial group scheme if and only if $T_{1, K}$ (resp. $T_{0, K}$ ) is trivial. From this (b) follows. The quotient group scheme $T / \operatorname{Ker}(\rho)$ exists and is a closed subgroup scheme of $\mathbb{G}_{m, A}^{m}$ that is of multiplicative type; see [SGA $3_{\text {II }}$ 1970, Exposé IX, Proposition 2.7(i) and Corollary 2.5]. As the fibers of $T / \operatorname{Ker}(\rho)$ are tori, we get that $T / \operatorname{Ker}(\rho)$ is a torus. Thus (c) holds.

The following lemma is only a variant of [Vasiu 2005a, Lemma 2.1].

Lemma 2.4. Suppose that $k=\bar{k}$. Let $H$ be a reductive group over $\operatorname{Spec} k$. Let $\mathfrak{n}$ be a nonzero ideal of $\mathrm{Lie}(H)$ which is a simple left $H$-module. We assume that there exists a maximal torus $T$ of $H$ such that we have $\operatorname{Lie}(T) \cap \mathfrak{n}=0$. Then $\operatorname{char}(k)=2$ and $H^{\mathrm{der}}$ has a normal, subgroup $F$ which is isomorphic to $\mathrm{SO}_{2 n+1, k}$ for some $n \in \mathbb{N}^{*}$ and for which we have an inclusion $\mathfrak{n} \subseteq \operatorname{Lie}(F)$.

Remark 2.4.1. If $\mathfrak{n}$ is assumed to be a restricted Lie subalgebra of $\operatorname{Lie}(H)$ (for instance, this holds if $\mathfrak{n}$ is the Lie algebra of a subgroup of $H$ ), then there exists a purely inseparable isogeny $H \rightarrow H / \mathfrak{n}$ (see [Borel 1991, Chapter V, Proposition 17.4]) and in this case Lemma 2.4 can be also deduced easily from [Prasad and Yu 2006, Lemma 2.2] applied to such isogenies with $H^{\text {ad }}$ absolutely simple. In this paper, Lemma 2.4 will be applied only in such situations in which $\mathfrak{n}$ is the Lie algebra of a subgroup of $H$.

Theorem 2.5. Let $f: G_{1} \rightarrow G_{2}$ be a homomorphism between group schemes over a scheme $Y$. We assume that $G_{1}$ is reductive, that $G_{2}$ is separated and of finite presentation, and that all fibers of $f$ are closed embeddings. Then $f$ is a closed embedding. 
Proof. As $G_{1}$ is of finite presentation over $Y$, the homomorphism $f$ is locally of finite type. As the fibers of $f$ are closed embeddings and thus monomorphisms, $f$ itself is a monomorphism (see [SGA $3_{\mathrm{I}} 1970$, Exposé $\mathrm{VI}_{\mathrm{B}}$, Corollary 2.11]). Thus the theorem follows from [SGA $3_{\text {II }}$ 1970, Exposé XVI, Corollary 1.5(a)].

Lemma 2.5.1. Let $G$ be an adjoint group scheme over an affine scheme $Y=\operatorname{Spec} A$. Let $\operatorname{Aut}(G)$ be the group scheme over $Y$ of automorphisms of $G$. Then the natural adjoint representation $\mathrm{Ad}: \operatorname{Aut}(G) \rightarrow \mathrm{GL}_{\mathrm{Lie}(G)}$ is a closed embedding.

Proof. To prove the lemma, we can work locally in the étale topology of $Y$ and therefore (see Proposition 2.3) we can assume that $G$ is split and that $Y$ is connected. We have a short exact sequence $1 \rightarrow G \rightarrow \operatorname{Aut}(G) \rightarrow C \rightarrow 1$ that splits (see [SGA $3_{\text {III }}$ 1970, Exposé XXIV, Theorem 1.3]), where $C$ is a finite, étale, constant group scheme over $Y$. Thus $G$ is the identity component of $\operatorname{Aut}(G)$, and $\operatorname{Aut}(G)$ is a finite disjoint union of right translates of $G$ via certain $Y$-valued points of $\operatorname{Aut}(G)$. If the fibers of Ad are closed embeddings, then the restriction of Ad to $G$ is a closed embedding (see Theorem 2.5), and thus also the restriction of Ad to any right translate of $G$ via a $Y$-valued point of $\operatorname{Aut}(G)$ is a closed embedding. The last two sentences imply that Ad is a closed embedding. Thus, to finish the proof, we are left to check that the fibers of Ad are closed embeddings. For this, we can assume that $A$ is an algebraically closed field.

As $G$ is adjoint and $A$ is a field, the restriction of Ad to $G$ is a closed embedding. Thus the representation Ad is a closed embedding if and only if each element $g \in \operatorname{Aut}(G)(A)$ that acts trivially on $\operatorname{Lie}(G)$ is trivial. We show that the assumption that there exists a nontrivial element $g$ leads to a contradiction. For this, we can assume that $G$ is absolutely simple and that $g$ is a nontrivial outer automorphism of $G$. Let $T$ be a maximal torus of a Borel subgroup $B$ of $G$ and let $n$ be the dimension of $T$.

For $t \in \operatorname{Lie}(T)$, let $C_{G}(t)$ be its centralizer in $G$; it is a subgroup of $G$ that contains $T$. In this paragraph we check that, as $G$ is adjoint, we can choose $t$ such that $C_{G}(t)^{0}=T$. We consider the root decomposition $\operatorname{Lie}(G)=\operatorname{Lie}(T) \bigoplus_{\alpha \in \Phi} \mathfrak{g}_{\alpha}$ with respect to $T$, where $\Phi$ is the root system of $G$ and where each $\mathfrak{g}_{\alpha}$ is a onedimensional $A$-vector space normalized by $T$. Let $\Delta$ be the basis for $\Phi$ such that we have $\operatorname{Lie}(B)=\operatorname{Lie}(T) \bigoplus_{\alpha \in \Delta} \mathfrak{g}_{\alpha}$. As $G$ is adjoint, $\Delta$ is a basis for the dual $A$-vector space $\operatorname{Lie}(T)^{*}$ (to be compared with [SGA $3_{\text {III }} 1970$, Exposé XXI, Definition 6.2.6 and Exposé XXII, Definition 4.3.3]). Thus for each root $\alpha \in \Delta$, $\operatorname{Ker}(\alpha)$ is an $A$-vector subspace of $\operatorname{Lie}(T)$ of dimension $n-1$. As each $\alpha \in \Phi$ is conjugate under the Weyl group of $\Phi$ (equivalently of $G$ ) to an element of $\Delta$ (see [Humphreys 1972, Chapter III, Section 10, Theorem]), we get that for each $\alpha \in \Delta$ its kernel $\operatorname{Ker}(\alpha)$ is an $A$-vector subspace of $\operatorname{Lie}(T)$ of dimension $n-1$. We choose $t \in \operatorname{Lie}(T) \backslash \bigcup_{\alpha \in \Phi} \operatorname{Ker}(\alpha)$. This implies that $\operatorname{Lie}\left(C_{G}(t)\right)=\operatorname{Lie}(T)$. From 
this and the fact that $T$ is a subgroup of $C_{G}(t)$, we get that $C_{G}(t)$ is a smooth group of dimension $n$ and therefore that $C_{G}(t)^{0}=T$.

As $g$ fixes $t$ and $\operatorname{Lie}(B)$, it normalizes both $C_{G}(t)^{0}=T$ and $B$. But it is well known that a nontrivial outer automorphism $g$ of $G$ that normalizes both $T$ and $B$ cannot fix $\operatorname{Lie}(B)$. Contradiction. Thus Ad is a closed embedding.

We follow the ideas of [Vasiu 1999, Proposition 3.1.2.1(c) and Remark 3.1.2.2(3)] in order to prove the next proposition.

Proposition 2.5.2. Let $V$ be a discrete valuation ring whose residue field is $k$. Let $Y=\operatorname{Spec} V$ and let $K:=K_{Y}$. Let $f: H_{1} \rightarrow H_{2}$ be a homomorphism between flat, finite type, affine group schemes over $Y$ such that $H_{1}$ is a reductive group scheme and the generic fiber $f_{K}: H_{1, K} \rightarrow H_{2, K}$ of $f$ is a closed embedding. We have:

(a) The subgroup scheme $\operatorname{Ker}\left(f_{k}: H_{1, k} \rightarrow H_{2, k}\right)$ of $H_{1, k}$ has a trivial intersection with each torus $T_{1, k}$ of $H_{1, k}$. In particular, $\operatorname{Lie}\left(\operatorname{Ker}\left(f_{k}\right)\right) \cap \operatorname{Lie}\left(T_{1, k}\right)=0$.

(b) The homomorphism $f$ is finite.

(c) If $\operatorname{char}(k)=2$, we assume that $H_{1, K}$ has no normal subgroup that is adjoint of isotypic $B_{n}$ Dynkin type for some $n \in \mathbb{N}^{*}$. Then $f$ is a closed embedding.

Proof. Let $\rho: H_{2} \hookrightarrow \mathrm{GL}_{M}$ be a closed embedding homomorphism, with $M$ a free $V$-module of finite rank (see [SGA 3 1 1970, Exposé $\mathrm{VI}_{\mathrm{B}}$, Remark 11.11.1]). To prove the proposition we can assume that $V$ is complete, that $k=\bar{k}$, and that $f_{K}: H_{1, K} \rightarrow H_{2, K}$ is an isomorphism. Let $H_{0, k}:=\operatorname{Ker}\left(f_{k}\right)$. We now show that the group scheme $H_{0, k} \cap T_{1, k}$ is trivial by adapting arguments from [Vasiu 1999, Remark 3.1.2.2(3) and proof of Lemma 3.1.6]. As $V$ is strictly henselian, the maximal torus $T_{1, k}$ of $H_{1, k}$ is split and (see Proposition 2.3) it lifts to a maximal torus $T_{1}$ of $H_{1}$. The restriction of $\rho \circ f$ to $T_{1}$ has a trivial kernel (as its fiber over Spec $K$ is trivial; see Lemma 2.3.2(b)) and therefore it is a closed embedding (see Lemma 2.3.2(c)). Thus the restriction of $f$ to $T_{1}$ is a closed embedding homomorphism $T_{1} \hookrightarrow H_{2}$. Therefore, the intersection $H_{0, k} \cap T_{1, k}$ is a trivial group scheme. Thus (a) holds.

We check (b). The identity component of the reduced scheme of $\operatorname{Ker}\left(f_{k}\right)$ is a reductive group that has 0 rank (see (a)) and therefore it is a trivial group. Thus $f$ is a quasifinite, birational morphism. From Zariski's main theorem (see [Grothendieck 1966, Theorem 8.12.6]) we get that $H_{1}$ is an open subscheme of the normalization $H_{2}^{\mathrm{n}}$ of $H_{2}$. Let $H_{3}$ be the smooth locus of $H_{2}^{\mathrm{n}}$ over Spec $V$; it is an open subscheme of $H_{2}^{\mathrm{n}}$ that contains $H_{1}$. As $H_{3}$ is an open subscheme of the affine scheme $H_{2}^{\mathrm{n}}$, it is a quasiaffine scheme.

As $H_{3}$ is smooth over $\operatorname{Spec} V$, the products $H_{3} \times \operatorname{Spec} V H_{2}^{\mathrm{n}}$ and $H_{2}^{\mathrm{n}} \times \operatorname{Spec} V H_{3}$ are smooth over $H_{2}^{\mathrm{n}}$ and thus are normal schemes. The product $H_{2}^{\mathrm{n}} \times \operatorname{Spec} V H_{2}^{\mathrm{n}}$ is a flat scheme over $\operatorname{Spec} V$ whose generic fiber is smooth over $\operatorname{Spec} K$. Its 
normalization $\left(H_{2}^{\mathrm{n}} \times \operatorname{Spec} V H_{2}^{\mathrm{n}}\right)^{\mathrm{n}}$ contains both $H_{3} \times \operatorname{Spec} V H_{2}^{\mathrm{n}}$ and $H_{2}^{\mathrm{n}} \times \operatorname{Spec} V H_{3}$ as open subschemes and is equipped with a finite surjective morphism given by $\left(H_{2}^{\mathrm{n}} \times \operatorname{Spec} V H_{2}^{\mathrm{n}}\right)^{\mathrm{n}} \rightarrow H_{2}^{\mathrm{n}} \times \operatorname{Spec} V H_{2}^{\mathrm{n}}$ whose generic fiber is an isomorphism. The product morphism $\mathrm{H}_{2} \times \operatorname{Spec~} V \mathrm{H}_{2} \rightarrow \mathrm{H}_{2}$ induces a natural product-type morphism $\Theta:\left(H_{2}^{\mathrm{n}} \times \operatorname{Spec} V H_{2}^{\mathrm{n}}\right)^{\mathrm{n}} \rightarrow H_{2}^{\mathrm{n}}$. Its restrictions to $H_{3} \times \operatorname{Spec} V H_{2}^{\mathrm{n}}$ and $H_{2}^{\mathrm{n}} \times \operatorname{Spec} V H_{3}$ induce product-type morphisms $H_{3} \times \operatorname{Spec} V H_{2}^{\mathrm{n}} \rightarrow H_{2}^{\mathrm{n}}$ and $H_{2}^{\mathrm{n}} \times \operatorname{Spec} V H_{3} \rightarrow H_{2}^{\mathrm{n}}$. This implies that for each valued point $z \in H_{2}^{\mathrm{n}}(V)$ it makes sense to speak about the left $z H_{3}$ and the right $H_{3} z$ translations of $H_{3}$ through $z$; they are smooth open subschemes of $H_{2}^{\mathrm{n}}$ and thus of $H_{3}$. This implies that $H_{3}(V)=H_{2}^{\mathrm{n}}(V)$ and that $\Theta$ restricts to a product morphism $H_{3} \times$ Spec $V H_{3} \rightarrow H_{3}$. The inverse automorphisms of the (Spec $V$ )-schemes $H_{1}$ and $H_{2}$ induce an inverse automorphism of the (Spec $V$ )scheme $H_{2}^{\mathrm{n}}$ which restricts to an inverse automorphism of the (Spec $V$ )-scheme $H_{3}$. With respect to its product morphism, its inverse automorphism, and its identity section inherited from $H_{1}$, the subscheme $H_{3}$ gets the structure of a (quasiaffine) group scheme over Spec $V$ that is of finite type.

As $V$ is complete, it is also excellent (see [Matsumura 1980, Section 34]). Thus the morphism $\mathrm{H}_{2}^{\mathrm{n}} \rightarrow \mathrm{H}_{2}$ is finite. The homomorphism $f$ is finite if and only if $H_{1}=H_{2}^{\mathrm{n}}$ and thus if and only if the set $H_{2}^{\mathrm{n}}(k) \backslash H_{1}(k)$ is empty. We show that the assumption $H_{1} \neq H_{2}^{\mathrm{n}}$ leads to a contradiction. Let $x \in H_{2}^{\mathrm{n}}(k) \backslash H_{1}(k)$. From [Vasiu 2012c, Lemma 4.1.5] applied to the completion of the local ring of $x$ in $H_{2}^{\mathrm{n}}$, we get that there exists a finite, flat discrete valuation ring extension $V^{\prime}$ of $V$ for which we have a valued point $z^{\prime} \in H_{2}^{\mathrm{n}}\left(V^{\prime}\right)$ that lifts $x$ (we recall that loc. cit. is only a local version of the global result [Grothendieck 1967, Corollary 17.16.2]). The flat (Spec $V^{\prime}$ )-scheme $H_{2, V^{\prime}}^{\mathrm{n}}$ might not be normal but we have $H_{1} \neq H_{2}^{\mathrm{n}}$ if and only if $H_{1, V^{\prime}} \neq H_{2, V^{\prime}}^{\mathrm{n}}$. Thus to reach a contradiction we can replace $V$ by $V^{\prime}$, and therefore we can assume that there exists a valued point $z \in H_{2}^{\mathrm{n}}(V)=H_{3}(V)$ which lifts $x$. As $x \in H_{2}^{\mathrm{n}}(k) \backslash H_{1}(k)$, we have $z \in H_{3}(V) \backslash H_{1}(V)$. As $H_{1}$ is a subgroup scheme of $H_{3}$, all fibers of the homomorphism $H_{1} \rightarrow H_{3}$ are closed. From this and Theorem 2.5 we get that $H_{1}$ is a closed subscheme of $H_{3}$. Thus, as $H_{3}$ is an integral scheme and as $H_{3, K}=H_{1, K}$, we get that $H_{1}=H_{3}$. This contradicts the fact that $z \in H_{3}(V) \backslash H_{1}(V)$. Thus (b) holds.

We check (c). We show that the assumption $\operatorname{Lie}\left(H_{0, k}\right) \neq 0$ leads to a contradiction. From Lemma 2.4 applied to $H_{1, k}$ and to any simple $H_{1, k}$-submodule of the left $H_{1, k}$-module $\operatorname{Lie}\left(H_{0, k}\right)$, we get that $\operatorname{char}(k)=2$ and that $H_{1, k}$ has a normal subgroup $H_{4, k}$ isomorphic to $\mathrm{SO}_{2 n+1, k}$ for some $n \in \mathbb{N}^{*}$. As $H_{4, k}$ is adjoint, we have a product decomposition $H_{1, k}=H_{4, k} \times{ }_{\text {Spec } k} H_{5, k}$ of reductive groups. It lifts (see [SGA $3_{\text {III }}$ 1970, Exposé XXIV, Proposition 1.21]) to a product decomposition $H_{1}=H_{4} \times \operatorname{Spec} V H_{5}$, where $H_{4}$ is isomorphic to $\mathrm{SO}_{2 n+1, V}$ and where $H_{5}$ is a reductive group scheme over $\operatorname{Spec} V$. This contradicts the extra hypothesis of (c). Thus we have $\operatorname{Lie}\left(H_{0, k}\right)=0$. Therefore, $H_{0, k}$ is a finite, étale, normal subgroup 
of $H_{1, k}$. But $H_{1, k}$ is connected and thus its action on $H_{0, k}$ via inner conjugation is trivial. Therefore, we have $H_{0, k} \leqslant Z\left(H_{1}\right)_{k} \leqslant T_{1, k}$. Thus $H_{0, k}=H_{0, k} \cap T_{1, k}$ is the trivial group; see (a). In other words, the homomorphism $f_{k}: H_{1, k} \rightarrow H_{2, k}$ is a closed embedding. Thus $f: H_{1} \rightarrow H_{2}$ is a closed embedding homomorphism; see Theorem 2.5.

Remark 2.5.3. See [Vasiu 2005b, Theorem 1.2(b)] and [Prasad and Yu 2006, Theorem 1.2] for two other proofs of Proposition 2.5.2(c).

\section{Lie algebras with perfect Killing forms}

Let $A$ be a commutative $\mathbb{Z}$-algebra. Let $\mathfrak{g}$ be a Lie algebra over $A$ which as an $A$-module is projective and finitely generated. In this section we will assume that the Killing form $\mathscr{K}_{\mathfrak{g}}$ on $\mathfrak{g}$ is perfect. Let $U_{\mathfrak{g}}$ be the enveloping algebra of $\mathfrak{g}$, i.e., the quotient of the tensor algebra $T_{\mathfrak{g}}$ of $\mathfrak{g}$ by the two-sided ideal of $T_{\mathfrak{g}}$ generated by the subset $\{x \otimes y-y \otimes x-[x, y] \mid x, y \in \mathfrak{g}\}$ of $T_{\mathfrak{g}}$. Let $Z\left(U_{\mathfrak{g}}\right)$ be the center of $U_{\mathfrak{g}}$. The categories of left $\mathfrak{g}$-modules and of left $U_{\mathfrak{g}}$-modules are canonically identified. We view $\mathfrak{g}$ as a left $\mathfrak{g}$-module via the adjoint representation ad: $\mathfrak{g} \rightarrow \mathfrak{g l}_{\mathfrak{g}}$; let ad: $U_{\mathfrak{g}} \rightarrow \operatorname{End}(\mathfrak{g})$ be the $A$-homomorphism corresponding to the left $\mathfrak{g}$-module $\mathfrak{g}$. We refer to [Cartan and Eilenberg 1956, Chapter XIII] for the cohomology groups $H^{i}(\mathfrak{g}, \mathfrak{v})$ of a left $\mathfrak{g}$-module $\mathfrak{v}$ (here $i \in \mathbb{N}$ ). We denote also by $\mathscr{K}_{\mathfrak{g}}: \mathfrak{g} \otimes_{A} \mathfrak{g} \rightarrow A$ the $A$-linear map defined by $\mathscr{K}_{\mathfrak{g}}: \mathfrak{g} \times \mathfrak{g} \rightarrow A$. Thus we have $\mathscr{K}_{\mathfrak{g}} \in\left(\mathfrak{g} \otimes_{A} \mathfrak{g}\right)^{*}=\mathfrak{g}^{*} \otimes_{A} \mathfrak{g}^{*}$. Let $\phi: \mathfrak{g} \stackrel{\sim}{\longrightarrow} \mathfrak{g}^{*}$ be the $A$-linear isomorphism defined naturally by $\mathcal{K}_{\mathfrak{g}}$. It induces an $A$-linear isomorphism $\phi^{-1} \otimes \phi^{-1}: \mathfrak{g}^{*} \otimes_{A} \mathfrak{g}^{*} \stackrel{\sim}{\longrightarrow} \mathfrak{g} \otimes_{A} \mathfrak{g}$. The image $\Omega$ of $\phi^{-1} \otimes \phi^{-1}\left(\mathcal{K}_{\mathfrak{g}}\right) \in \mathfrak{g} \otimes_{A} \mathfrak{g} \subseteq T_{\mathfrak{g}}$ in $U_{\mathfrak{g}}$ is called the Casimir element of the adjoint representation ad: $\mathfrak{g} \rightarrow \mathfrak{g l}_{\mathfrak{g}}$.

Lemma 3.1. For the Casimir element $\Omega \in U_{\mathfrak{g}}$ the following four properties hold:

(a) if the A-module $\mathfrak{g}$ is free and if $\left\{x_{1}, \ldots, x_{m}\right\}$ and $\left\{y_{1}, \ldots, y_{m}\right\}$ are two A-bases for $\mathfrak{g}$ such that for all $i, j \in\{1, \ldots, m\}$ we have $\mathcal{K}_{\mathfrak{g}}\left(x_{i} \otimes y_{j}\right)=\delta_{i j}$, then $\Omega$ is the image of the element $\sum_{i=1}^{m} x_{i} \otimes y_{i}$ of $T_{\mathfrak{g}}$ in $U_{\mathfrak{g}}$;

(b) we have $\Omega \in Z\left(U_{\mathfrak{g}}\right)$;

(c) the Casimir element $\Omega$ is fixed by the group of Lie automorphisms of $\mathfrak{g}$ (i.e., if $\sigma: U_{\mathfrak{g}} \stackrel{\sim}{\longrightarrow} U_{\mathfrak{g}}$ is the A-algebra automorphism induced by a Lie algebra automorphism $\sigma: \mathfrak{g} \sim \mathfrak{\longrightarrow}$, then we have $\sigma(\Omega)=\Omega$ );

(d) the Casimir element $\Omega$ acts identically on $\mathfrak{g}\left(\right.$ i.e., $\left.\operatorname{ad}(\Omega)=1_{\mathfrak{g}}\right)$.

Proof. Parts (a) and (b) are proved in [Bourbaki 1989, Chapter I, Section 3.7, Proposition 11]. Strictly speaking, loc. cit. is stated over a field but its proof applies over any commutative $\mathbb{Z}$-algebra. This is so, as the essence of the proof is [Bourbaki 1989, Chapter I, Section 3.5, Example 2] which is worked out over 
any commutative $\mathbb{Z}$-algebra. In particular, [Bourbaki 1989, Chapter I, Section 3.5, Example 3] can be easily stated over a commutative $\mathbb{Z}$-algebra (by involving a perfect invariant bilinear form over a commutative $\mathbb{Z}$-algebra instead of a nondegenerate invariant bilinear form over a field). We recall here that $\mathscr{K}_{\mathfrak{g}}$ is $\mathfrak{g}$-invariant, i.e., for all $a, b, c \in \mathfrak{g}$ we have an identity $\mathscr{K}_{\mathfrak{g}}(\operatorname{ad}(a)(b), c)+\mathscr{K}_{\mathfrak{g}}(b, \operatorname{ad}(a)(c))=0$ (see [Bourbaki 1989, Chapter I, Section 3.6, (13) and Proposition 8]), and this is the very essence of (b).

To check (c) and (d), we can assume that the $A$-module $\mathfrak{g}$ is free. Let $\left\{x_{1}, \ldots, x_{m}\right\}$ and $\left\{y_{1}, \ldots, y_{m}\right\}$ be two $A$-bases for $\mathfrak{g}$ as in (a). Thus $\Omega$ is the image of $\sum_{i=1}^{m} x_{i} \otimes y_{i}$ in $U_{\mathfrak{g}}$. Therefore, $\sigma(\Omega)$ is the image of $\sum_{i=1}^{m} \sigma\left(x_{i}\right) \otimes \sigma\left(y_{i}\right)$ in $U_{\mathfrak{g}}$. As we have $\mathcal{K}_{\mathfrak{g}}\left(\sigma\left(x_{i}\right), \sigma\left(y_{j}\right)\right)=\delta_{i j}$ for $i, j \in\{1, \ldots, m\}$, from (a) we get that the image of $\sum_{i=1}^{m} \sigma\left(x_{i}\right) \otimes \sigma\left(y_{i}\right) \in T_{\mathfrak{g}}$ in $U_{\mathfrak{g}}$ is $\Omega$. Thus $\sigma(\Omega)=\Omega$.

We check (d). Let $z, w \in \mathfrak{g}$. We write $\operatorname{ad}(z) \circ \operatorname{ad}(w)\left(x_{i}\right)=\sum_{j=1}^{m} a_{j i} x_{j}$, with the $a_{j i} \mathrm{~s}$ in $A$. Using the $\mathfrak{g}$-invariance of $\mathscr{K}_{\mathfrak{g}}$ we compute

$\mathscr{K}_{\mathfrak{g}}(\operatorname{ad}(\Omega)(z), w)$

$$
\begin{aligned}
& =\mathscr{K}_{\mathfrak{g}}\left(\sum_{i=1}^{m} \operatorname{ad}\left(x_{i}\right) \circ \operatorname{ad}\left(y_{i}\right)(z), w\right)=-\sum_{i=1}^{m} \mathscr{K}_{\mathfrak{g}}\left(\operatorname{ad}\left(y_{i}\right)(z), \operatorname{ad}\left(x_{i}\right)(w)\right) \\
& =\sum_{i=1}^{m} \mathscr{K}_{\mathfrak{g}}\left(\operatorname{ad}(z)\left(y_{i}\right), \operatorname{ad}\left(x_{i}\right)(w)\right)=-\sum_{i=1}^{m} \mathcal{K}_{\mathfrak{g}}\left(y_{i}, \operatorname{ad}(z) \circ \operatorname{ad}\left(x_{i}\right)(w)\right) \\
& =\sum_{i=1}^{m} \mathscr{K}_{\mathfrak{g}}\left(y_{i}, \operatorname{ad}(z) \circ \operatorname{ad}(w)\left(x_{i}\right)\right)=\sum_{i, j=1}^{m} a_{j i} \delta_{j i}=\sum_{i=1}^{m} a_{i i}=\mathscr{K}_{\mathfrak{g}}(z, w),
\end{aligned}
$$

where the last equality is due to the very definition of $\mathscr{K}_{\mathfrak{g}}$. This implies that, for each $z \in \mathfrak{g}$, we have $\operatorname{ad}(\Omega)(z)-z \in \operatorname{Ker}\left(\mathscr{K}_{\mathfrak{g}}\right)=0$. Thus $\operatorname{ad}(\Omega)(z)=z$, i.e., (d) holds.

Fact 3.2. Let $i \in \mathbb{N}$. Let $\mathfrak{v}$ be a left $\mathfrak{g}$-module on which $\Omega$ acts identically. Then the cohomology group $H^{i}(\mathfrak{g}, \mathfrak{v})$ is trivial.

Proof. We have an identity $H^{i}(\mathfrak{g}, \mathfrak{v})=\operatorname{Ext}_{U_{\mathfrak{g}}}^{i}(A, \mathfrak{v})$ of $Z\left(U_{\mathfrak{g}}\right)$-modules; see [Cartan and Eilenberg 1956, Chapter XIII, Sections 2 and 8]. As $\Omega \in Z\left(U_{\mathfrak{g}}\right)$ acts trivially on $A$ and identically on $\mathfrak{v}$, the group $\Omega \operatorname{Ext}_{U_{\mathfrak{g}}}^{i}(A, \mathfrak{v})$ is on one hand trivial and on the other hand equal to $\operatorname{Ext}_{U_{\mathfrak{g}}}^{i}(A, \mathfrak{v})$. Therefore, $\operatorname{Ext}_{U_{\mathfrak{g}}}^{i}(A, \mathfrak{v})=0$, and $H^{i}(\mathfrak{g}, \mathfrak{v})=0$.

Theorem 3.3. We recall that the Killing form $\mathscr{K}_{\mathfrak{g}}$ on $\mathfrak{g}$ is perfect. Then the group scheme Aut $(\mathfrak{g})$ over Spec A of Lie automorphisms of $\mathfrak{g}$ is smooth and locally of finite presentation.

Proof. To check this, we can assume that the $A$-module $\mathfrak{g}$ is free. The group scheme $\operatorname{Aut}(\mathfrak{g})$ is a closed subgroup scheme of $\mathrm{GL}_{\mathfrak{g}}$ defined by a finitely generated ideal of the ring of functions of $\mathrm{GL}_{\mathfrak{g}}$. Thus $\operatorname{Aut}(\mathfrak{g})$ is of finite presentation. Therefore, to show that $\operatorname{Aut}(\mathfrak{g})$ is smooth over $\operatorname{Spec} A$, it suffices to show that, for each 
affine morphism Spec $B \rightarrow \operatorname{Spec} A$ and for each ideal $\mathfrak{j}$ of $B$ such that $\mathfrak{j}^{2}=0$, the restriction map $\operatorname{Aut}(\mathfrak{g})(B) \rightarrow \operatorname{Aut}(\mathfrak{g})(B / \mathfrak{j})$ is onto (see [Bosch et al. 1990, Chapter 2, Section 2.2, Proposition 6]). So as not to introduce extra notation by repeatedly tensoring with $B$ over $A$, we will assume that $B=A$. Thus $j$ is an ideal of $A$ and we have to show that the restriction map $\operatorname{Aut}(\mathfrak{g})(A) \rightarrow \operatorname{Aut}(\mathfrak{g})(A / \mathfrak{j})$ is onto.

Let $\bar{\sigma}: \mathfrak{g} / \mathfrak{j g} \stackrel{\sim}{\longrightarrow} \mathfrak{g} / \mathfrak{j g}$ be a Lie automorphism. Let $\sigma_{0}: \mathfrak{g} \stackrel{\sim}{\rightarrow} \mathfrak{g}$ be an $A$-linear automorphism that lifts $\bar{\sigma}$. Let $\mathfrak{j} \mathfrak{g}_{\bar{\sigma}}$ be the left $\mathfrak{g}$-module which as an $A$-module is $\mathfrak{j g}$ and whose left $\mathfrak{g}$-module structure is defined as follows: if $x \in \mathfrak{g}$, then $x$ acts on $\mathfrak{j} \mathfrak{g}_{\bar{\sigma}}$ in the same way as $\operatorname{ad}(\bar{\sigma}(x))$ (equivalently, as $\left.\operatorname{ad}\left(\sigma_{0}(x)\right)\right)$ acts on the $A$-module $\mathfrak{j} \mathfrak{g}=\mathfrak{j g}_{\bar{\sigma}}$; this makes sense as $\mathfrak{j}^{2}=0$. Let $\theta: \mathfrak{g} \times \mathfrak{g} \rightarrow \mathfrak{j} \mathfrak{g}_{\bar{\sigma}}$ be the alternating map defined by the rule

$$
\theta(x, y):=\left[\sigma_{0}(x), \sigma_{0}(y)\right]-\sigma_{0}([x, y]) \text { for all } x, y \in \mathfrak{g} .
$$

We check that $\theta$ is a 2-cocycle, i.e., for all $x, y, z \in \mathfrak{g}$ we have an identity

$$
\begin{aligned}
& d \theta(x, y, z) \\
& \quad:=x(\theta(y, z))-y(\theta(x, z))+z(\theta(x, y))-\theta([x, y], z)+\theta([x, z], y)-\theta([y, z], x) \\
& \quad=0 .
\end{aligned}
$$

Substituting (1) in the definition of $d \theta$, we get that the expression $d \theta(x, y, z)$ is a sum of 12 terms which can be divided into three groups as follows. The first group contains the three terms

$$
-\sigma_{0}([[x, y], z]), \quad \sigma_{0}([[x, z], y]), \quad-\sigma_{0}([[y, z], x]) ;
$$

their sum is 0 due to the Jacobi identity and the fact that $\sigma_{0}$ is an $A$-linear map. The second group contains the six terms

$$
\begin{array}{rrr}
{\left[\sigma_{0}(x), \sigma_{0}[y, z]\right],} & -\left[\sigma_{0}(x), \sigma_{0}[y, z]\right], & {\left[\sigma_{0}(y), \sigma_{0}[x, z]\right],} \\
-\left[\sigma_{0}(y), \sigma_{0}[x, z]\right], & {\left[\sigma_{0}(z), \sigma_{0}[x, y]\right],} & -\left[\sigma_{0}(z), \sigma_{0}[x, y]\right] ;
\end{array}
$$

obviously their sum is 0 . The third group contains the three terms

$$
\left[\sigma_{0}(x),\left[\sigma_{0}(y), \sigma_{0}(z)\right]\right], \quad-\left[\sigma_{0}(y),\left[\sigma_{0}(x), \sigma_{0}(z)\right]\right], \quad\left[\sigma_{0}(z),\left[\sigma_{0}(x), \sigma_{0}(y)\right]\right] ;
$$

their sum is 0 due to the Jacobi identity. Thus, indeed, $d \theta=0$.

As $\Omega$ (i.e., ad $(\Omega)$ ) acts identically on $\mathfrak{g}$ (see Lemma 3.1(d)), it also acts identically on $\mathfrak{j} \mathfrak{g}$. But $\Omega$ modulo $\mathfrak{j}$ is fixed by the Lie automorphism $\bar{\sigma}$ of $\mathfrak{g} / \mathfrak{j} \mathfrak{g}$; see Lemma 3.1(c). Thus $\Omega$ also acts identically on the left $\mathfrak{g}$-module $\mathfrak{j} \mathfrak{g}_{\bar{\sigma}}$. From this and Fact 3.2 we get that $H^{2}\left(\mathfrak{g}, \mathfrak{j} \mathfrak{g}_{\bar{\sigma}}\right)=0$. Thus $\theta$ is the coboundary of a 1 -cochain $\delta: \mathfrak{g} \rightarrow \mathfrak{j} \mathfrak{g}_{\bar{\sigma}}$, i.e., we have

$$
\theta(x, y)=x(\delta(y))-y(\delta(x))-\delta([x, y]) \quad \text { for all } x, y \in \mathfrak{g} .
$$


Let $\sigma: \mathfrak{g} \stackrel{\sim}{\longrightarrow} \mathfrak{g}$ be the $A$-linear isomorphism defined by the rule $\sigma(x):=\sigma_{0}(x)-\delta(x)$; here $\delta(x)$ is an element of the $A$-module $\mathfrak{j} \mathfrak{g}=\mathfrak{j} \mathfrak{g}_{\bar{\sigma}}$. Due to formulas (1) and (2), we compute

$$
\begin{aligned}
\sigma([x, y]) & =\sigma_{0}([x, y])-\delta([x, y]) \\
& =\left[\sigma_{0}(x), \sigma_{0}(y)\right]-\theta(x, y)-\delta([x, y]) \\
& =\left[\sigma_{0}(x), \sigma_{0}(y)\right]-x(\delta(y))+y(\delta(x)) \\
& =\left[\sigma_{0}(x), \sigma_{0}(y)\right]-\operatorname{ad}(\bar{\sigma}(x))(\delta(y))+\operatorname{ad}(\bar{\sigma}(y))(\delta(x)) \\
& =\left[\sigma_{0}(x), \sigma_{0}(y)\right]-\operatorname{ad}\left(\sigma_{0}(x)\right)(\delta(y))+\operatorname{ad}\left(\sigma_{0}\right)(y)(\delta(x)) \\
& =\left[\sigma_{0}(x), \sigma_{0}(y)\right]-\left[\sigma_{0}(x), \delta(y)\right]+\left[\sigma_{0}(y), \delta(x)\right] \\
& =\left[\sigma_{0}(x)-\delta(x), \sigma_{0}(y)-\delta(y)\right]-[\delta(x), \delta(y)] \\
& =[\sigma(x), \sigma(y)]-[\delta(x), \delta(y)] \\
& =[\sigma(x), \sigma(y)],
\end{aligned}
$$

where the last identity follows from $\mathfrak{j}^{2}=0$. Therefore, $\sigma$ is a Lie automorphism of $\mathfrak{g}$ that lifts the Lie automorphism $\bar{\sigma}$ of $\mathfrak{g} / \mathfrak{j} \mathfrak{g}$. Thus the restriction map $\operatorname{Aut}(\mathfrak{g})(A) \rightarrow$ $\operatorname{Aut}(\mathfrak{g})(A / \mathfrak{j})$ is onto.

3.4. Proof of Theorem 1.2. The functor $\mathscr{L}_{Y}$ is faithful; see Lemma 2.5.1. Thus to prove Theorem 1.2 it suffices to show that $\mathscr{L}_{Y}$ is surjective on objects and that $\mathscr{L}_{Y}$ is fully faithful. To check this, as Adj-perf $f_{Y}$ and Lie-perf $f_{Y}$ are groupoids on sets and as $\mathscr{L}_{Y}$ is faithful, we can assume that $Y=\operatorname{Spec} A$ is affine. Thus to finish the proof it suffices to check the following three properties:

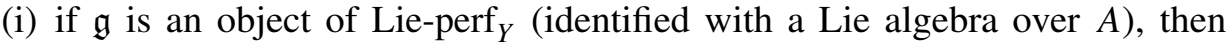
there exists a unique open subgroup scheme $\operatorname{Aut}(\mathfrak{g})^{0}$ of $\operatorname{Aut}(\mathfrak{g})$ which is an adjoint group scheme over $Y$ and whose Lie algebra is the Lie subalgebra ad( $(\mathfrak{g})$ of $\mathfrak{g l}_{\mathfrak{g}}$ (therefore $\mathfrak{g}=\operatorname{ad}(\mathfrak{g})$ is the image through $\mathscr{L}_{Y}$ of the object $\operatorname{Aut}(\mathfrak{g})^{0}$ of

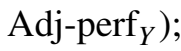

(ii) the group scheme $\operatorname{Aut}\left(\operatorname{Aut}(\mathfrak{g})^{0}\right)$ of automorphisms of $\operatorname{Aut}(\mathfrak{g})^{0}$ is $\operatorname{Aut}(\mathfrak{g})$ acting on $\operatorname{Aut}(\mathfrak{g})^{0}$ via inner conjugation (therefore $\left.\operatorname{Aut}(\mathfrak{g})(A)=\operatorname{Aut}\left(\operatorname{Aut}(\mathfrak{g})^{0}\right)(A)\right)$;

(iii) if $G$ and $H$ are two objects of $\operatorname{Adj-perf}_{Y}$ such that $\operatorname{Lie}(G)=\operatorname{Lie}(H)$, then $G$ and $H$ are isomorphic.

To check the first two properties, we can assume that the $A$-module $\mathfrak{g}$ is free and of rank $m \in \mathbb{N}^{*}$. Let $k$ be the residue field of an arbitrary point $y \in Y$. It is well known that the Lie algebra $\operatorname{Lie}\left(\operatorname{Aut}(\mathfrak{g})_{k}\right)$ is the Lie algebra of derivations of $\mathfrak{g}_{k}:=\mathfrak{g} \otimes_{A} k$. As this fact plays a key role in this paper, we include a proof of it. The tangent space of $\operatorname{Aut}(\mathfrak{g})_{k}$ at the identity element is identified with the set of 
automorphisms $a$ of $\mathfrak{g}_{k} \otimes_{k} k[\varepsilon] /\left(\varepsilon^{2}\right)$, which modulo $\bar{\varepsilon}=\varepsilon+\left(\varepsilon^{2}\right)$ are the identity automorphism of $\mathfrak{g}_{k}$. We can write each such automorphism as

$$
a=1_{\mathfrak{g}_{k} \otimes_{k} k[\varepsilon] /\left(\varepsilon^{2}\right)}+D_{a} \otimes \bar{\varepsilon},
$$

where $D_{a}$ is a $k$-linear endomorphism of $\mathfrak{g}_{k}$. The condition that $a$ respects the Lie bracket (i.e., $a([u, v] \otimes 1)=[a(u \otimes 1), a(v \otimes 1)]$ for all $\left.u, v \in \mathfrak{g}_{k}\right)$ is equivalent to the condition that $D_{a}$ is a derivation of $\mathfrak{g}_{k}$. The association $a \mapsto D_{a}$ identifies the tangent space of $\operatorname{Aut}(\mathfrak{g})_{k}$ at the identity element with the $k$-vector space of derivations of $\mathfrak{g}_{k}$. Under this identification, the Lie bracket of $a$ with an automorphism $b$ of $\mathfrak{g}_{k} \otimes_{k} k\left[\varepsilon_{1}\right] /\left(\varepsilon_{1}^{2}\right)$, which modulo $\bar{\varepsilon}_{1}=\varepsilon_{1}+\left(\varepsilon_{1}^{2}\right)$ is the identity automorphism of $\mathfrak{g}_{k}$, is the derivation of $\mathfrak{g}_{k}$ which corresponds to the automorphism

$$
a b a^{-1} b^{-1}=1_{\mathfrak{g}_{k} \otimes k\left[\varepsilon \varepsilon_{1}\right] /\left(\varepsilon^{2} \varepsilon_{1}^{2}\right)}+\left[D_{a}, D_{b}\right] \bar{\varepsilon} \bar{\varepsilon}_{1}
$$

of $\mathfrak{g}_{k} \otimes_{k} k\left[\varepsilon \varepsilon_{1}\right] /\left(\varepsilon^{2} \varepsilon_{1}^{2}\right)$ and thus is the Lie bracket $\left[D_{a}, D_{b}\right]\left(\varepsilon_{1}\right.$ is used here instead of $\varepsilon$ so that this last part makes sense). Therefore, $\operatorname{Lie}\left(\operatorname{Aut}(\mathfrak{g})_{k}\right)$ is the Lie algebra of derivations of $\mathfrak{g}_{k}$.

As the Killing form $\mathscr{K}_{\mathfrak{g}_{k}}$ is perfect, one argues as in [Humphreys 1972, Chapter II, Section 5.3, Theorem] that each derivation of $\mathfrak{g}_{k}$ is an inner derivation. Thus we have $\operatorname{Lie}\left(\operatorname{Aut}(\mathfrak{g})_{k}\right)=\operatorname{ad}(\mathfrak{g}) \otimes_{A} k$. As the group scheme $\operatorname{Aut}(\mathfrak{g})$ over $Y$ is smooth and locally of finite presentation (see Theorem 3.3), from [SGA 3 $3_{\mathrm{I}}$ 1970, Exposé VI $\mathrm{V}_{\mathrm{B}}$, Corollary 4.4] we get that there exists a unique open subgroup scheme $\operatorname{Aut}(\mathfrak{g})^{0}$ of $\operatorname{Aut}(\mathfrak{g})$ whose fibers are connected. The fibers of $\operatorname{Aut}(\mathfrak{g})^{0}$ are open-closed subgroups of the fibers of $\operatorname{Aut}(\mathfrak{g})$ and thus are affine.

Let $N_{k}$ be a smooth, connected, unipotent, normal subgroup of $\operatorname{Aut}(\mathfrak{g})_{k}^{0}$. The Lie algebra $\operatorname{Lie}\left(N_{k}\right)$ is a nilpotent ideal of $\operatorname{Lie}\left(\operatorname{Aut}(\mathfrak{g})_{k}^{0}\right)=\operatorname{ad}(\mathfrak{g}) \otimes_{A} k$. Thus

$$
\operatorname{Lie}\left(N_{k}\right) \subseteq \operatorname{Ker}\left(\mathscr{K}_{\operatorname{Lie}\left(\operatorname{Aut}(\mathfrak{g})_{k}^{0}\right)}\right)=\operatorname{Ker}\left(\mathscr{K}_{\mathrm{ad}(\mathfrak{g}) \otimes_{A} k}\right) ;
$$

see [Bourbaki 1989, Chapter I, Section 4.4, Proposition 6(b)]. As the Killing form $\mathscr{K}_{\mathrm{ad}(\mathfrak{g}) \otimes_{A} k}$ is perfect, we get $\operatorname{Lie}\left(N_{k}\right)=0$. Thus $N_{k}$ is the trivial subgroup of $\operatorname{Aut}(\mathfrak{g})_{k}^{0}$, and therefore the unipotent radical of $\operatorname{Aut}(\mathfrak{g})_{k}^{0}$ is trivial. Thus $\operatorname{Aut}(\mathfrak{g})_{k}^{0}$ is an affine, connected, smooth group over Spec $k$ whose unipotent radical is trivial. Therefore, $\operatorname{Aut}(\mathfrak{g})_{k}^{0}$ is a reductive group over Spec $k$; see [Borel 1991, Chapter IV, Section 11.21]. As $\operatorname{Lie}\left(\operatorname{Aut}(\mathfrak{g})_{k}^{0}\right)=\operatorname{ad}(\mathfrak{g}) \otimes_{A} k$ has trivial center, the $\operatorname{group} \operatorname{Aut}(\mathfrak{g})_{k}^{0}$ is semisimple. Thus the smooth group scheme $\operatorname{Aut}(\mathfrak{g})^{0}$ of finite presentation over $Y$ has semisimple fibers. Therefore, $\operatorname{Aut}(\mathfrak{g})^{0}$ is a semisimple group scheme over $Y$; see [SGA $3_{\text {II }}$ 1970, Exposé XVI, Theorem 5.2(ii)]. As $Z\left(\operatorname{Aut}(\mathfrak{g})^{0}\right)_{k}$ acts trivially on $\operatorname{Lie}\left(\operatorname{Aut}(\mathfrak{g})_{k}^{0}\right)=\operatorname{ad}(\mathfrak{g}) \otimes_{A} k$ and as $Z\left(\operatorname{Aut}(\mathfrak{g})^{0}\right)_{k}$ is a subgroup of $\operatorname{Aut}(\mathfrak{g})_{k}$, the group $Z\left(\operatorname{Aut}(\mathfrak{g})^{0}\right)_{k}$ is trivial. This implies that the finite, flat group scheme $Z\left(\operatorname{Aut}(\mathfrak{g})^{0}\right)$ is trivial and thus $\operatorname{Aut}(\mathfrak{g})^{0}$ is an adjoint group scheme. 
The Lie subalgebras $\operatorname{Lie}\left(\operatorname{Aut}(\mathfrak{g})^{0}\right)$ and $\operatorname{ad}(\mathfrak{g})$ of $\mathfrak{g l}_{\mathfrak{g}}$ are free $A$-submodules of the Lie subalgebra $\mathfrak{l}$ of $\mathfrak{g l}_{\mathfrak{g}}$ formed by derivations of $\mathfrak{g}$. As, for each point $y$ of $Y$, we have $\operatorname{Lie}\left(\operatorname{Aut}(\mathfrak{g})_{k}^{0}\right)=\operatorname{ad}(\mathfrak{g}) \otimes_{A} k=\mathfrak{l} \otimes_{A} k$, the Lie subalgebra $\mathfrak{l}$ is locally generated by either $\operatorname{Lie}\left(\operatorname{Aut}(\mathfrak{g})^{0}\right)$ or ad $(\mathfrak{g})$. We easily get the identities $\operatorname{Lie}\left(\operatorname{Aut}(\mathfrak{g})^{0}\right)=\operatorname{ad}(\mathfrak{g})=\mathfrak{l}$.

The group scheme $\operatorname{Aut}(\mathfrak{g})$ acts via inner conjugation on $\operatorname{Aut}(\mathfrak{g})^{0}$. As we have $\operatorname{Lie}\left(\operatorname{Aut}(\mathfrak{g})^{0}\right)=\operatorname{ad}(\mathfrak{g})$ and as $\operatorname{Aut}(\mathfrak{g})$ is a closed subgroup scheme of $\mathrm{GL}_{\mathfrak{g}}$, the inner conjugation homomorphism $\operatorname{Aut}(\mathfrak{g}) \rightarrow \operatorname{Aut}\left(\operatorname{Aut}(\mathfrak{g})^{0}\right)$ has trivial kernel. As $\operatorname{Aut}\left(\operatorname{Aut}(\mathfrak{g})^{0}\right)$ is a closed subgroup scheme of $\operatorname{Aut}\left(\operatorname{Lie}\left(\operatorname{Aut}(\mathfrak{g})^{0}\right)\right)=\operatorname{Aut}(\operatorname{ad}(\mathfrak{g}))(\operatorname{see}$ Lemma 2.5.1), we can identify naturally $\operatorname{Aut}\left(\operatorname{Aut}(\mathfrak{g})^{0}\right)$ with a closed subgroup scheme of $\operatorname{Aut}(\mathfrak{g})$. From the last two sentences, we get that $\operatorname{Aut}\left(\operatorname{Aut}(\mathfrak{g})^{0}\right)=\operatorname{Aut}(\mathfrak{g})$. Thus both properties (i) and (ii) hold.

To check that property (iii) holds, let $\mathfrak{g}=\operatorname{Lie}(G)=\operatorname{Lie}(H)$. It suffices to show that $G$ and $H$ are identified with $\operatorname{Aut}(\mathfrak{g})^{0}$. We will work only with $G$. The adjoint representation $G \rightarrow \mathrm{GL}_{\mathfrak{g}}$ factors as composite closed embedding homomorphisms $G \rightarrow \operatorname{Aut}(\mathfrak{g})^{0} \rightarrow \operatorname{Aut}(\mathfrak{g}) \rightarrow \mathrm{GL}_{\mathfrak{g}}$ (see Lemma 2.5.1 and [SGA 3 III 1970, Exposé XXIV, Theorem 1.3]). We get a closed embedding homomorphism $G \rightarrow \operatorname{Aut}(\mathfrak{g})^{0}$ between adjoint group schemes that have the same Lie algebra $\mathfrak{g}$ (see also property (i)). By reasons of dimensions, the geometric fibers of the closed embedding homomorphism $G \rightarrow \operatorname{Aut}(\mathfrak{g})^{0}$ are isomorphisms, and therefore $G \rightarrow \operatorname{Aut}(\mathfrak{g})^{0}$ is an isomorphism. Thus property (iii) holds as well.

The next proposition details the range of applicability of Theorem 1.2.

Proposition 3.5. (a) We recall that $k$ is a field. Let $H$ be a nontrivial semisimple group over Spec $k$. Then the Killing form $\mathscr{K}_{\operatorname{Lie}(H)}$ is perfect if and only if the following two conditions hold:

(i) either char $(k)$ equals 0 or char $(k)$ is an odd prime $p$ and $H^{\text {ad }}$ has no simple factor of isotypic $A_{p n-1}, B_{p n+\frac{1-p}{2}}, C_{p n-1}$, or $D_{p n+1}$ Dynkin type (here $n \in \mathbb{N}^{*}$ );

(ii) if $\operatorname{char}(k)=3$ (resp. $\operatorname{char}(k)=5)$, then $H^{\mathrm{ad}}$ has no simple factor of isotypic $E_{6}, E_{7}, E_{8}, F_{4}, G_{2}$ (resp. isotypic $\left.E_{8}\right)$ Dynkin type.

(b) If $\mathscr{K}_{\mathrm{Lie}(H)}$ is perfect, then the central isogenies $H^{\mathrm{sc}} \rightarrow H \rightarrow H^{\mathrm{ad}}$ are étale; thus, by identifying tangent spaces at identity elements, $\operatorname{Lie}\left(H^{\mathrm{sc}}\right)=\operatorname{Lie}(H)=\operatorname{Lie}\left(H^{\mathrm{ad}}\right)$.

Proof. We can assume that $k=\bar{k}$ and that $\operatorname{tr} \operatorname{deg}(k)<\infty$. If $\operatorname{char}(k)=0$, then $\operatorname{Lie}(H)$ is a semisimple Lie algebra over $k$ and therefore the proposition follows from [Humphreys 1972, Chapter II, Section 5.1, Theorem]. Thus we can assume $\operatorname{char}(k)$ is a prime $p \in \mathbb{N}^{*}$. If conditions (i) and (ii) hold, then $p$ does not divide the order of the finite group scheme $Z\left(H^{\mathrm{sc}}\right)=\operatorname{Ker}\left(H^{\mathrm{sc}} \rightarrow H^{\text {ad }}\right)$ (see [Bourbaki 2002, Plates I to IX]) and therefore (a) implies (b).

Let $W(k)$ be the ring of $p$-typical Witt vectors with coefficients in $k$. Let $H_{W(k)}$ be a semisimple group scheme over Spec $W(k)$ that lifts $H$; see [SGA $3_{\text {III }}$ 1970, Exposé 
XXIV, Proposition 1.21]. Then $\operatorname{Lie}\left(H_{W(k)}^{\mathrm{sc}}\right)\left[\frac{1}{p}\right]=\operatorname{Lie}\left(H_{W(k)}\right)\left[\frac{1}{p}\right]=\operatorname{Lie}\left(H_{W(k)}^{\mathrm{ad}}\right)\left[\frac{1}{p}\right]$. This implies that:

(iii) the Killing form $\mathscr{K}_{\mathrm{Lie}\left(H_{W(k)}\right)}$ is the composite of the natural $W(k)$-linear map $\operatorname{Lie}\left(H_{W(k)}\right) \times \operatorname{Lie}\left(H_{W(k)}\right) \rightarrow \operatorname{Lie}\left(H_{W(k)}^{\mathrm{ad}}\right) \times \operatorname{Lie}\left(H_{W(k)}^{\mathrm{ad}}\right)$ with $\mathcal{K}_{\operatorname{Lie}\left(H_{W(k)}^{\mathrm{ad}}\right)} ;$

(iv) the Killing form $\mathscr{K}_{\operatorname{Lie}\left(H_{W(k)}^{\text {sc }}\right)}$ is the composite of the natural $W(k)$-linear map $\operatorname{Lie}\left(H_{W(k)}^{\mathrm{sc}}\right) \times \operatorname{Lie}\left(H_{W(k)}^{\mathrm{sc}}\right) \rightarrow \operatorname{Lie}\left(H_{W(k)}\right) \times \operatorname{Lie}\left(H_{W(k)}\right)$ with $\mathcal{K}_{\operatorname{Lie}\left(H_{W(k)}\right)}$.

We prove (a). We have $\operatorname{Ker}\left(\operatorname{Lie}(H) \rightarrow \operatorname{Lie}\left(H^{\mathrm{ad}}\right)\right) \subseteq \operatorname{Ker}\left(\mathscr{K}_{\operatorname{Lie}(H)}\right)$; see property (iii). If $\mathscr{K}_{\operatorname{Lie}(H)}$ is perfect, then $\operatorname{Ker}\left(\operatorname{Lie}(H) \rightarrow \operatorname{Lie}\left(H^{\text {ad }}\right)\right)=0$ and therefore $\operatorname{Lie}(H)=\operatorname{Lie}\left(H^{\text {ad }}\right)$. Thus to prove (a) we can assume that $H=H^{\text {ad }}$ is adjoint. Even more, to prove (a) we can also assume that the adjoint group $H$ is simple; let $b$ be the Lie type of $H$. If $b$ is not of classical Lie type, then $\mathscr{K}_{\mathrm{Lie}(H)}$ is perfect if and only if either $p>5$ or $p=5$ and $b \neq E_{8}$ (see [Humphreys 1995, Table, p. 49]). Thus to prove (a), we can assume that $b$ is a classical Lie type. We fix a morphism $\operatorname{Spec} \mathbb{C} \rightarrow \operatorname{Spec} W(k)$.

Suppose that b is either $A_{n}$ or $C_{n}$. By the standard trace form on $\operatorname{Lie}\left(H^{\mathrm{sc}}\right)$ (resp. $\operatorname{Lie}\left(H_{W(k)}^{\mathrm{sc}}\right)$ or $\operatorname{Lie}\left(H_{\mathbb{C}}^{\mathrm{sc}}\right)$ ) we mean the trace form $\mathscr{T}\left(\right.$ resp. $\mathscr{T}_{W(k)}$ or $\left.\mathscr{T}_{\mathbb{C}}\right)$ associated to the faithful representation of $H^{\mathrm{sc}}$ (resp. $H_{W(k)}^{\mathrm{sc}}$ or $H_{\mathbb{C}}^{\mathrm{sc}}$ ) of rank $n+1$ if $b=A_{n}$ and of rank $2 n$ if $b=C_{n}$. We have $\mathscr{K}_{\operatorname{Lie}\left(H_{\mathbb{C}}^{\text {sc }}\right)}=2(n+1) \mathscr{T}_{\mathbb{C}}$; see [Helgason 1978, Chapter III, Section 8, (5) and (22)]. This identity implies that we also have $\mathscr{K}_{\mathrm{Lie}\left(H_{W(k)}^{\mathrm{sc}}\right)}=2(n+1) \mathscr{T}_{W(k)}$ and thus $\mathscr{K}_{\mathrm{Lie}\left(H^{\mathrm{sc}}\right)}=2(n+1) \mathscr{T}$. If $p$ does not divide $2(n+1)$, then $\operatorname{Lie}\left(H^{\mathrm{sc}}\right)=\operatorname{Lie}(H)$ and it is well known that $\mathscr{T}$ is perfect; thus $\mathscr{K}_{\mathrm{Lie}\left(H^{\mathrm{sc}}\right)}=\mathscr{K}_{\mathrm{Lie}(H)}=2(n+1) \mathscr{T}$ is perfect. Suppose that $p$ divides $2(n+1)$. This implies that $\mathscr{K}_{\mathrm{Lie}\left(H^{\mathrm{sc}}\right)}$ is the trivial bilinear form on $\operatorname{Lie}\left(H^{\mathrm{sc}}\right)$. From this and property (iv) we get that the restriction of $\mathscr{K}_{\operatorname{Lie}(H)}$ to $\operatorname{Im}\left(\operatorname{Lie}\left(H^{\mathrm{sc}}\right) \rightarrow \operatorname{Lie}(H)\right)$ is trivial. As $\operatorname{dim}_{k}\left(\operatorname{Lie}(H) / \operatorname{Im}\left(\operatorname{Lie}\left(H^{\mathrm{sc}}\right) \rightarrow \operatorname{Lie}(H)\right)\right)=1$ and $\operatorname{dim}_{k}(\operatorname{Lie}(H)) \geq 3$, we easily get that $\mathscr{K}_{\mathrm{Lie}(H)}$ is degenerate.

Suppose that $b=B_{n}$ (resp. $b=D_{n}$ with $n \geq 4$ ). If $p>2$, then we have $\operatorname{Lie}\left(H^{\mathrm{sc}}\right)=\operatorname{Lie}(H)$. Moreover, using [Helgason 1978, Chapter III, Section 8, (11) and (15)], as in the previous paragraph we argue that $\mathscr{K}_{\operatorname{Lie}(H)}$ is perfect if $p$ does not divide $2(2 n-1)$ (resp. if $p$ does not divide $2(n-1))$ and is degenerate if $p$ divides $2 n-1$ (resp. if $p$ divides $2(n-1)$ ).

We are left to show that $\mathscr{K}_{\mathrm{Lie}(H)}$ is degenerate if $p=2$ and $b=B_{n}$. The group $H$ is (isomorphic to) the SO-group of the quadratic form $x_{0}^{2}+x_{1} x_{n+1}+\cdots+x_{n} x_{2 n}$ on $W:=k^{2 n+1}$. Let $\left\{e_{i, j} \mid i, j \in\{0,1, \ldots, n\}\right.$ be the standard $k$-basis for $\mathfrak{g l}_{W}$. The direct sum $\mathfrak{n}_{n}:=\bigoplus_{i=1}^{2 n} k e_{0, i}$ is a nilpotent ideal of Lie $(H)$; see [Borel 1991, Chapter V, Section 23.6]. Thus $\mathfrak{n}_{n} \subseteq \operatorname{Ker}\left(\mathscr{K}_{\operatorname{Lie}(H)}\right)$, by [Bourbaki 1989, Chapter I, Section 4.4, Proposition 6(b)] applied to the adjoint representation of $\operatorname{Lie}(H)$. Therefore, $\mathscr{K}_{\mathrm{Lie}(H)}$ is degenerate. 
We conclude that $\mathscr{K}_{\mathrm{Lie}(H)}$ is perfect if and only if both conditions (i) and (ii) hold. Therefore, (a) (and thus also (b)) holds.

Remark 3.6. Let $A$ and $\mathfrak{g}$ be as in the beginning of this section.

(a) Let $p \in \mathbb{N}^{*}$ be a prime. Suppose that $A$ is an algebraically closed field of characteristic $p$. Let $G$ be an adjoint group over $\operatorname{Spec} A$ such that $\mathfrak{g}=\operatorname{Lie}(G)$; see Theorem 1.2. We have $p \neq 2$; see Proposition 3.5. Let $G_{\mathbb{Z}}$ be the unique (up to isomorphism) split, adjoint group scheme over Spec $\mathbb{Z}$ such that $G$ is the pullback of $G_{\mathbb{Z}}$ to Spec $A$; see [SGA $3_{\text {III }}$ 1970, Exposé XXV, Corollary 1.3]. We have $\mathfrak{g}=\operatorname{Lie}\left(G_{\mathbb{Z}}\right) \otimes_{\mathbb{Z}} A$, i.e., $\mathfrak{g}$ has a canonical model $\operatorname{Lie}\left(G_{\mathbb{Z}}\right)$ over $\mathbb{Z}$. For $p>7$, this result was obtained in [Curtis 1957, Section 5, Theorem]. For $p>3$, this result was obtained by Seligman [1967, Chapter II, Section 10], Mills [1957], Mills and Seligman [1957], and Block and Zassenhaus [1964]. For $p=3$, this result was obtained in [Brown 1969, Theorem 4.1]. It seems to us that the fact that $p \neq 2$ (i.e., that all Killing forms of finite dimensional Lie algebras over fields of characteristic 2 are degenerate) is new.

(b) Let $B \rightarrow A$ be an epimorphism of commutative $\mathbb{Z}$-algebras whose kernel $\mathfrak{j}$ is a nilpotent ideal. Then $\mathfrak{g}$ has, up to isomorphisms, a unique lift to a Lie algebra over $B$ which as a $B$-module is projective and finitely generated. One can prove this statement using cohomological methods as in the proof of Theorem 3.3. The statement also follows from Theorem 1.2 and the fact that $\operatorname{Aut}(\mathfrak{g})^{0}$ has, up to isomorphisms, a unique lift to an adjoint group scheme over $\operatorname{Spec} B$ (this can be easily checked at the level of torsors of adjoint group schemes; see [SGA $3_{\text {III }}$ 1970, Exposé XXIV, Corollaries 1.17 and 1.18]).

Corollary 3.7. Let $\mathrm{Sc}-\mathrm{perf}_{Y}$ be the category whose objects are simply connected semisimple group schemes over $Y$ with the property that their Lie algebra $\mathrm{O}_{Y^{-}}$ modules have perfect Killing forms and whose morphisms are isomorphisms of group schemes. Then the functor $\mathscr{L}_{Y}^{\text {sc }}: \mathrm{Sc}^{-\operatorname{perf}_{Y}} \rightarrow \operatorname{Lie} \operatorname{perf}_{Y}$, which associates to a morphism $f: G \stackrel{\sim}{\longrightarrow} H$ of Sc-perf $_{Y}$ the morphism $d f: \operatorname{Lie}(G) \stackrel{\sim}{\longrightarrow} \operatorname{Lie}(H)$ of Lie-perf $_{Y}$ which is the differential of $f$, is an equivalence of categories.

Proof. The functor $\mathscr{L}_{Y}^{\text {sc }}$ is the composite of the canonical ('division by the centers') functor $\mathscr{L}_{Y}: \mathrm{Sc}$-perf ${ }_{Y} \rightarrow \operatorname{Adj}$-perf ${ }_{Y}$ with $\mathscr{L}_{Y}$; the functor $\mathscr{L}_{Y}$ makes sense (see Proposition 3.5(b)) and it is an equivalence of categories. Thus the corollary follows from Theorem 1.2.

Corollary 3.8. The category Lie-perf $_{Y}$ has a nonzero object if and only if $Y$ is a nonempty Spec $\mathbb{Z}\left[\frac{1}{2}\right]$-scheme.

Proof. The 'if' part is implied by the fact that an $\mathfrak{s l}_{2}$ Lie algebra $\mathscr{O}_{Y}$-module has perfect Killing form. The 'only if' part follows from the relation $p \neq 2$ of Remark 3.6(a). 


\section{Proof of Theorem 1.4}

In this section we prove Theorem 1.4. See Sections 4.1 and 4.2 for the proofs of Theorem 1.4(a) and Theorem 1.4(b) (respectively). In Remark 4.3 we point out that the hypotheses of Theorem 1.4 are indeed needed in general. We will use the notation presented in Section 1.

4.1. Proof of Theorem 1.4(a). To prove Theorem 1.4 we can assume $Y$ is also integral. Let $K:=K_{Y}$ be a field. If $H$ is a reductive group scheme over $Y$, then we have $\mathscr{D}(H)=\mathscr{D}\left(H_{U}\right)$ and thus the uniqueness parts of Theorem 1.4 follow from Proposition 2.2(b). Let $\mathfrak{l}$ be the Lie algebra $\widehat{O}_{Y}$-module which extends $\operatorname{Lie}\left(G_{U}\right)$.

We prove Theorem 1.4(a). Due to the uniqueness part, to prove Theorem 1.4(a) we can assume $Y=\operatorname{Spec} A$ is also local and strictly henselian. Let $\mathfrak{g}:=\mathfrak{l}(Y)$ be the Lie algebra over $A$ of global sections of $\mathfrak{l}$.

As $U$ is connected, based on [SGA $3_{\text {III }}$ 1970, Exposé XXII, Proposition 2.8] we can speak about the split, adjoint group scheme $S$ over $Y$ of the same Lie type as all geometric fibers of $G_{U}$. Let $\mathfrak{s}:=\operatorname{Lie}(S)$. Let $\operatorname{Aut}(S)$ be the group scheme over $Y$ of automorphisms of $S$. We have a short exact sequence $1 \rightarrow S \rightarrow \operatorname{Aut}(S) \rightarrow C \rightarrow 1$, where $C$ is a finite, étale, constant group scheme over $Y$ (see [SGA $3_{\text {III }} 1970$, Exposé XXIV, Theorem 1.3]). Let $\gamma \in H^{1}\left(U, \operatorname{Aut}(S)_{U}\right)$ be the class that defines the form $G_{U}$ of $S_{U}$.

We recall that $\mathrm{GL}_{\mathfrak{g}}$ and $\mathrm{GL}_{\mathfrak{s}}$ are the reductive group schemes over $Y$ of linear automorphisms of $\mathfrak{g}$ and $\mathfrak{s}$ (respectively). The adjoint representations define closed embedding homomorphisms $j_{U}: G_{U} \hookrightarrow \mathrm{GL}_{\mathfrak{g}, U}$ and $i: S \hookrightarrow \mathrm{GL}_{\mathfrak{s}}$ and, moreover, $i$ extends naturally to a closed embedding homomorphism $\operatorname{Aut}(S) \hookrightarrow \mathrm{GL}_{\mathfrak{s}}$; see Lemma 2.5.1. Let $\delta \in H^{1}\left(U,\left(\mathrm{GL}_{\mathfrak{s}, U}\right)\right)$ be the image of $\gamma$ via the homomorphism $\operatorname{Aut}(S)_{U} \hookrightarrow \mathrm{GL}_{\mathfrak{s}, U}$.

We recall that the quotient sheaf for the faithfully flat topology of $Y$ of the action of $S$ on $\mathrm{GL}_{\mathfrak{s}}$ via right translations is representable by a $Y$-scheme $\mathrm{GL}_{\mathfrak{s}} / S$ that is affine and that causes $\mathrm{GL}_{\mathfrak{s}}$ to be a right torsor of $S$ over $\mathrm{GL}_{\mathfrak{s}} / S$ (see [ColliotThélène and Sansuc 1979, Corollary 6.12]). Thus $\mathrm{GL}_{\mathfrak{s}} / S$ is a smooth, affine $Y$-scheme. The finite, étale, constant group scheme $C$ acts naturally (from the right) on $\mathrm{GL}_{\mathfrak{s}} / S$ and this action is free (see Lemma 2.5.1). From [SGA $3_{\mathrm{I}}$ 1970, Exposé V, Theorem 4.1] we get that the quotient $Y$-scheme $\left(\mathrm{GL}_{\mathfrak{s}} / S\right) / C$ is affine and that the quotient epimorphism $\mathrm{GL}_{\mathfrak{s}} / S \rightarrow\left(\mathrm{GL}_{\mathfrak{s}} / S\right) / C$ is a finite étale cover. Thus $\left(\mathrm{GL}_{\mathfrak{s}} / S\right) / C$ is a smooth, affine scheme over $Y$ that represents the quotient sheaf for the faithfully flat topology of $Y$ of the action of $\operatorname{Aut}(S)$ on $\mathrm{GL}_{\mathfrak{s}}$ via right translations. From constructions we get that $\mathrm{GL}_{\mathfrak{s}}$ is a right torsor of $\operatorname{Aut}(S)$ over $\mathrm{GL}_{\mathfrak{s}} / \operatorname{Aut}(S):=\left(\mathrm{GL}_{\mathfrak{s}} / S\right) / C$.

The twist of $i_{U}$ via the class $\gamma$ is $j_{U}$. This implies that the class $\delta$ defines the torsor that parametrizes isomorphisms between the pullbacks to $U$ of the vector group 
schemes over $Y$ defined by $\mathfrak{s}$ and $\mathfrak{g}$. Therefore, as the $A$-modules $\mathfrak{s}$ and $\mathfrak{g}$ are isomorphic (being free and of equal ranks), the class $\delta$ is trivial. Thus $\gamma$ is the coboundary of a class in $H^{0}\left(U, \mathrm{GL}_{\mathfrak{s}, U} / \operatorname{Aut}(S)_{U}\right)$. But $H^{0}\left(U, \mathrm{GL}_{\mathfrak{s}, U} / \operatorname{Aut}(S)_{U}\right)$ equals $H^{0}\left(Y, \mathrm{GL}_{\mathfrak{s}} / \operatorname{Aut}(S)\right)$ (see Proposition 2.2(b)) and therefore $\gamma$ is the restriction of a class in $H^{1}(Y, \operatorname{Aut}(S))$. As $Y$ is strictly henselian, each class in $H^{1}(Y, \operatorname{Aut}(S))$ is trivial. Thus $\gamma$ is the trivial class. Therefore, the group schemes $G_{U}$ and $S_{U}$ are isomorphic. Thus $G_{U}$ extends to an adjoint group scheme $G$ over $Y$ isomorphic to $S$.

4.2. Proof of Theorem 1.4(b). Let $\eta: \bar{K} \rightarrow U$ be the geometric point of $U$ which is the composite of the natural morphisms $\operatorname{Spec} \bar{K} \rightarrow \operatorname{Spec} K$ and Spec $K \rightarrow U$. We denote also by $\eta: \bar{K} \rightarrow Y$ the resulting geometric point of $Y$. As $Y$ (resp. $U$ ) is normal and locally noetherian, from [SGA $3_{\text {II }}$ 1970, Exposé X, Theorems 5.16 and 7.1] we get that there exists an antiequivalence of categories between the category of tori over $Y$ (resp. $U$ ) and the category of continuous $\pi_{1}(Y, \eta)$-representations (resp. continuous $\pi_{1}(U, \eta)$-representations) on free $\mathbb{Z}$-modules of finite rank. As the pair $(Y, Y \backslash U)$ is quasipure, we have a canonical identification $\pi_{1}(U, \eta)=\pi(Y, \eta)$. From the last two sentences we get that there exists a unique torus $H^{\text {ab }}$ over $Y$ which extends $H_{U}^{\mathrm{ab}}$.

Let $H^{\text {ad }}$ be the adjoint group scheme over $Y$ that extends $H_{U}^{\text {ad }}$; see Theorem 1.4(a). Let $F \rightarrow H^{\text {ad }} \times{ }_{Y} H^{\text {ab }}$ be the central isogeny over $Y$ that extends the central isogeny $H_{K} \rightarrow H_{K}^{\mathrm{ad}} \times{ }_{\text {Spec } K} H_{K}^{\mathrm{ab}}$; see Lemma 2.3.1(a). Both $F_{U}$ and $H_{U}$ are the normalization of $H_{U}^{\mathrm{ab}} \times_{U} H_{U}^{\mathrm{ad}}$ in $H_{K}$; see Lemma 2.3.1(b). Thus $H_{U}=F_{U}$ extends uniquely to a reductive group scheme $H:=F$ over $Y$ (see the first paragraph of Section 4.1 for the uniqueness part).

Remark 4.3. (a) Let $Y_{1} \rightarrow Y$ be a finite, nonétale morphism between normal, noetherian, integral Spec $\mathbb{Z}_{(2)}$-schemes such that there exists an open subscheme $U$ of $Y$ with the properties that: (i) $Y \backslash U$ has codimension in $Y$ at least 2, and (ii) $Y_{1} \times_{Y} U \rightarrow U$ is a Galois cover of degree 2. Let $H_{U}$ be the rank-1 nonsplit torus over $U$ that splits over $Y_{1} \times_{Y} U$. Then $H_{U}$ does not extend to a smooth, affine group scheme over $Y$. If, moreover, $Y=\operatorname{Spec} A$ is an affine $\operatorname{Spec} \mathbb{F}_{2}$-scheme, then we have $\operatorname{Lie}\left(H_{U}\right)(U)=A$ and therefore $\operatorname{Lie}\left(H_{U}\right)$ extends to a Lie algebra $O_{Y}$-module which as an $\mathrm{O}_{Y}$-module is free. Thus the quasipure part of the hypotheses of Theorem 1.4(b) is needed in general.

(b) Suppose that $Y=\operatorname{Spec} A$ is local, strictly henselian, regular, and of dimension $n \geq 3$. Let $K:=K_{Y}$. Let $d \in \mathbb{N}^{*}$ be such that there exists an $A$-submodule $M$ of $K^{d}$ that contains $A^{d}$, is of finite type, is not free, and satisfies the identity $M=\bigcap_{V \in \mathscr{D}(Y)} M \otimes_{A} V$ (inside $M \otimes_{A} K$ ). A typical example (communicated to us by Serre) is $d=n-1$ and $M \stackrel{\sim}{\rightarrow} \operatorname{Coker}(f)$, where the $A$-linear map $f: A \rightarrow A^{n}$ takes 1 to an $n$-tuple $\left(x_{1}, \ldots, x_{n}\right) \in A^{n}$ of regular parameters of $A$. 
Let $\mathscr{F}$ be the coherent $O_{Y}$-module defined by $M$. Let $U$ be an open subscheme of $Y$ such that $Y \backslash U$ has codimension in $Y$ at least 2 and the restriction $\mathscr{F}_{U}$ of $\mathscr{F}_{F}$ to $U$ is a locally free $O_{U}$-module. Let $H_{U}$ be the reductive group scheme over $U$ of linear automorphisms of $\mathscr{F}_{U}$. We recall the reason why the assumption that $H_{U}$ extends to a reductive group scheme $H$ over $Y$ leads to a contradiction. The group scheme $H$ is isomorphic to $\mathrm{GL}_{d, A}$ (as $A$ is strictly henselian), and therefore there exists a free $A$-submodule $L$ of $K^{d}$ of rank $d$ such that $H=\mathrm{GL}_{L}$. As $A$ is a unique factorization domain (being local and regular), it is easy to see that there exists an element $f \in K$ such that the identity $M \otimes_{A} V=f L \otimes_{A} V$ holds for each $V \in \mathscr{D}(Y)$. This implies that $M=f L$. Thus $M$ is a free $A$-module. Contradiction.

As $H_{U}$ does not extend to a reductive group scheme over $Y$ and as the pair $(Y, Y \backslash U)$ is quasipure, from Section 4.2 we get that $H_{U}^{\text {ad }}$ also does not extend to an adjoint group scheme over $Y$. Thus the Lie part of the hypotheses of Theorem 1.4(a) is needed in general.

\section{Extending homomorphisms via schematic closures}

In this section we prove two results on extending homomorphisms of reductive group schemes via taking (normalizations of) schematic closures. Proposition 5.1 complements Theorem 1.4(b) and Proposition 2.5.2, and Proposition 5.2 refines [Vasiu 1999, Lemma 3.1.6].

Proposition 5.1. Let $Y$ be a normal, noetherian, integral scheme. Let $K:=K_{Y}$. Let $U$ be an open subscheme of $Y$ such that the codimension of $Y \backslash U$ in $Y$ is at least 2. Let $H_{U}$ be a reductive group scheme over $U$ and let $G$ be a reductive group scheme over $Y$. We assume we have a finite homomorphism $\rho_{U}: H_{U} \rightarrow G_{U}$ whose generic fiber over Spec $K$ is a closed embedding. We assume that one of the following two properties holds:

(i) $H_{U}$ extends to a reductive group scheme $H$ over $Y$, or

(ii) $Y=\operatorname{Spec} R$ is a local regular scheme of dimension 2 (thus $U$ is the complement in $Y$ of the closed point of $Y$ ).

Then the following three properties hold:

(a) There exists a unique reductive group scheme $H$ over $Y$ which extends $H_{U}$.

(b) The homomorphism $\rho_{U}$ extends uniquely to a finite homomorphism $\rho: H \rightarrow G$ between reductive group schemes over $Y$.

(c) If there exists a point of $Y \backslash U$ of characteristic 2, we assume that $H_{K}$ has no normal subgroup that is adjoint of isotypic $B_{n}$ Dynkin type for some $n \in \mathbb{N}^{*}$. Then $\rho: H \rightarrow G$ is a closed embedding. 
Proof. If (i) holds, then the uniqueness of $H_{U}$ follows from Proposition 2.2(b). Thus to prove (a) we can assume that property (ii) holds. As (ii) holds, the pair $(Y, Y \backslash U)$ is quasipure (see Section 1) and the Lie algebra $O_{U}$-module Lie $\left(H_{U}\right)$ extends to a Lie algebra $\mathrm{O}_{Y}$-module which is a free $\mathrm{O}_{Y}$-module (by Proposition 2.2(c) and the fact that $Y$ is local). Thus the hypotheses of Theorem 1.4(b) hold, and therefore from Theorem 1.4(b) we get that there exists a unique reductive group scheme $H$ over $Y$ that extends $H_{U}$. Thus (a) holds.

To prove (b) and (c) we can assume that $Y=\operatorname{Spec} R$ is an affine scheme. We write $H=\operatorname{Spec} R_{H}$ and $G=\operatorname{Spec} R_{G}$. As $\mathscr{D}(H)=\mathscr{D}\left(H_{U}\right)$ and $\mathscr{D}(G)=\mathscr{D}\left(G_{U}\right)$, from Proposition 2.2(a) we get that $R_{H}$ and $R_{G}$ are the $R$-algebras of global functions of $H_{U}$ and $G_{U}$ (respectively). Let $R_{G} \rightarrow R_{H}$ be the $R$-homomorphism defined by $\rho_{U}$ and let $\rho: H \rightarrow G$ be the morphism of $Y$-schemes it defines. The morphism $\rho$ is a homomorphism, as it is so generically. To check that $\rho$ is finite, we can assume that $R$ is complete. Thus $R_{H}$ and $R_{G}$ are excellent rings; see [Matsumura 1980, Section 34]. Therefore, the normalization $H^{\prime}=\operatorname{Spec} R_{H^{\prime}}$ of the schematic closure of $H_{K}$ in $G$ is a finite, normal $G$-scheme.

The identity components of the reduced geometric fibers of $\rho$ are trivial groups; see Proposition 2.5.2(a) or (b). Thus $\rho$ is a quasifinite morphism. From Zariski's main theorem (see [Grothendieck 1966, Theorem 8.12.6]) we get that $H$ is an open subscheme of $H^{\prime}$. But from Proposition 2.5.2(b) we get that the morphism $H \rightarrow H^{\prime}$ satisfies the valuative criterion of properness with respect to discrete valuation rings which contain $R$. As each local ring of $H^{\prime}$ is dominated by such a discrete valuation ring, we get that the morphism $H \rightarrow H^{\prime}$ is surjective. Therefore, the open, surjective morphism $H \rightarrow H^{\prime}$ is an isomorphism. Thus $\rho$ is finite, i.e., (b) holds.

We prove (c). The pullback of the homomorphism $\rho: H \rightarrow G$ via each dominant morphism Spec $V \rightarrow Y$, with $V$ a discrete valuation ring, is a closed embedding (see Proposition 2.5.2(c)). This implies that the fibers of $\rho$ are closed embeddings. Thus the homomorphism $\rho$ is a closed embedding; see Theorem 2.5.

We have the following refinement of [Vasiu 1999, Lemma 3.1.6].

Proposition 5.2. Let $G$ be a reductive group scheme over a reduced, affine scheme $Y=\operatorname{Spec} A$. Let $K$ be a localization of $A$. Let $s \in \mathbb{N}^{*}$. For $j \in\{1, \ldots, s\}$ let $G_{j, K}$ be a reductive, closed subgroup scheme of $G_{K}$. We assume that the group subschemes $G_{j, K}$ commute among themselves and that either

(i) the direct sum $\bigoplus_{j=1}^{s} \operatorname{Lie}\left(G_{j, K}\right)$ is a Lie subalgebra of $\operatorname{Lie}\left(G_{K}\right)$, or

(ii) $s=2, G_{1, K}$ is a torus, and $G_{2, K}$ is a semisimple group scheme.

Then the closed subgroup scheme $G_{0, K}$ of $G_{K}$ generated by the group subschemes $G_{j, K}$ exists and is reductive. Moreover:

(a) If condition (i) holds, then $\operatorname{Lie}\left(G_{0, K}\right)=\bigoplus_{j=1}^{s} \operatorname{Lie}\left(G_{j, K}\right)$. 
(b) We assume that for each $j \in\{1, \ldots, s\}$ the schematic closure $G_{j}$ of $G_{j, K}$ in $G$ is a reductive group scheme over $Y$. Then the schematic closure $G_{0}$ of $G_{0, K}$ in $G$ is a reductive, closed subgroup scheme of $G$.

Proof. Let $\Lambda$ be the category whose objects $\mathrm{Ob}(\Lambda)$ are finite subsets of $K$ and whose morphisms are the inclusions of subsets. For $\alpha \in \mathrm{Ob}(\Lambda)$, let $K_{\alpha}$ be the $\mathbb{Z}$-subalgebra of $K$ generated by $\alpha$ and let $A_{\alpha}:=A \cap K_{\alpha}$. We have $K=$ ind $\lim _{\alpha \in \mathrm{Ob}(\Lambda)} K_{\alpha}$ and $A=$ ind $\lim _{\alpha \in \mathrm{Ob}(\Lambda)} A_{\alpha}$. The reductive group schemes $G_{j, K}$ are of finite presentation. Based on this and [Grothendieck 1966, Theorems 8.8.2 and 8.10.5], one gets that there exists a $\beta \in \mathrm{Ob}(\Lambda)$ such that each $G_{j, K}$ is the pullback of a closed subgroup scheme $G_{j, K_{\beta}}$ of $G_{K_{\beta}}$. For $\alpha \supseteq \beta$, the set $C(\alpha)$ of points of Spec $K_{\alpha}$ with the property that the fibers over them of all morphisms $G_{j, K_{\alpha}} \rightarrow$ Spec $K_{\alpha}$ are (geometrically) connected is a constructible set (see [Grothendieck 1966, Theorem 9.7.7]). We have proj $\lim _{\alpha \in \mathrm{Ob}(\Lambda)} C(\alpha)=\operatorname{Spec} K$. From this and [Grothendieck 1966, Theorem 8.5.2], we get that there exists a $\beta_{1} \in \mathrm{Ob}(\Lambda)$ such that $\beta_{1} \supseteq \beta$ and $C\left(\beta_{1}\right)=\operatorname{Spec} K_{\beta_{1}}$. Thus, by replacing $\beta$ with $\beta_{1}$, we can assume that the fibers of all morphisms $G_{j, K_{\beta}} \rightarrow$ Spec $K_{\beta}$ are connected. A similar argument shows that, by enlarging $\beta$, we can assume that all morphisms $G_{j, K_{\beta}} \rightarrow$ Spec $K_{\beta}$ are smooth and that their fibers are reductive groups (the role of [Grothendieck 1966, Theorem 9.7.7] being replaced by [Grothendieck 1966, Corollary 9.9.5] applied to the ${\mathcal{O}_{G_{j, K_{\alpha}}}}$-module $\operatorname{Lie}\left(G_{j, K_{\alpha}}\right)$ and by [SGA $3_{\text {III }}$ 1970, Exposé XIX, Corollary 2.6]). Thus each $G_{j, K_{\beta}}$ is a reductive closed subgroup scheme of $G_{K_{\beta}}$. The smooth group schemes $G_{j, K_{\beta}}$ commute among themselves, as this is so after pullback through the dominant morphism Spec $K \rightarrow$ Spec $K_{\beta}$. By enlarging $\beta$, we can also assume that either condition (i) or condition (ii) holds for the $G_{j, K_{\beta}}$ s and that $K_{\beta}$ is a localization of $A_{\beta}$. By replacing $A$ with the local ring of $\operatorname{Spec} A_{\beta}$ dominated by $A$, to prove the proposition we can assume that $A$ is a localization of a reduced, finitely generated $\mathbb{Z}$-algebra.

Using induction on $s \in \mathbb{N}^{*}$, it suffices to prove the proposition for $s=2$. Moreover, we can assume that $K=K_{Y}$. For the sake of flexibility, in what follows we will only assume that $A$ is a reduced, noetherian $\mathbb{Z}$-algebra; thus $K$ is a finite product of fields. As all the statements of the proposition are local for the étale topology of $Y$, it suffices to prove the proposition under the extra assumption that $G_{1}$ and $G_{2}$ are split (see Proposition 2.3). Let $C_{K}:=G_{1, K} \cap G_{2, K}$ be a closed subgroup scheme of $G_{j, K}$ that commutes with $G_{j, K}, j \in\{1,2\}$. The Lie algebra Lie $\left(C_{K}\right)$ is included in $\operatorname{Lie}\left(G_{1, K}\right) \cap \operatorname{Lie}\left(G_{2, K}\right)$ and therefore it is trivial if condition (i) holds. Thus if condition (i) holds, then $C_{K}$ is a finite, étale, closed subgroup scheme of $Z\left(G_{j, K}\right)$. If condition (ii) holds, then $C_{K}$ is a closed subgroup scheme of both $G_{1, K}=Z\left(G_{1, K}\right)$ and $Z\left(G_{2, K}\right)$ and thus (as $K$ is a finite product of fields) it is a finite group scheme of multiplicative type.

Let $C$ be the schematic closure of $C_{K}$ in $G$. Let $T_{j}$ be a maximal torus of $G_{j}$. 
We have

$$
C_{K} \leqslant Z\left(G_{1, K}\right) \cap Z\left(G_{2, K}\right) \leqslant T_{1, K} \cap T_{2, K} \leqslant G_{1, K} \cap G_{2, K}=C_{K}
$$

and thus $C_{K}=T_{1, K} \cap T_{2, K}$. Let $T_{1} \times_{Y} T_{2} \rightarrow G$ be the product homomorphism. The kernel $\mathfrak{K}$ of this product homomorphism is a group scheme over $Y$ of multiplicative type (see Lemma 2.3.2(a)) isomorphic to $T_{1} \cap T_{2}$. But $\mathfrak{K}_{K} \stackrel{\sim}{\longrightarrow} C_{K}$ is a finite group scheme over Spec $K$ and therefore $\mathfrak{K}$ is a finite, flat group scheme over $Y$ of multiplicative type (see Lemma 2.3.2(b)). Thus $T_{1} \cap T_{2}$ is a finite, flat group scheme over $Y$. From this, the identity $C_{K}=\left(T_{1} \cap T_{2}\right)_{K}$, and the definition of $C$ we get that $C=T_{1} \cap T_{2}$. We conclude that $C$ is a finite, flat group scheme over $Y$ of multiplicative type contained in the center of both $G_{1}$ and $G_{2}$. We embed $C$ in $G_{1} \times_{Y} G_{2}$ via the natural embedding $C \hookrightarrow G_{1}$ and via the composite of the inverse isomorphism $C \stackrel{\sim}{\longrightarrow} C$ with the natural embedding $C \hookrightarrow G_{2}$. Let $G_{1,2}:=\left(G_{1} \times{ }_{Y} G_{2}\right) / C$ be a reductive group scheme over $Y$. We have a natural product homomorphism $q: G_{1,2} \rightarrow G$ whose pullback to Spec $K$ can be identified with the closed embedding homomorphism $G_{0, K} \hookrightarrow G_{K}$. Therefore, $G_{0, K}$ is a reductive group scheme over Spec $K$. Moreover, if condition (i) holds, then as $C_{K}$ is étale we have natural identities

$$
\operatorname{Lie}\left(G_{1, K}\right) \oplus \operatorname{Lie}\left(G_{2, K}\right)=\operatorname{Lie}\left(G_{1,2, K}\right)=\operatorname{Lie}\left(G_{0, K}\right) .
$$

Thus (a) holds. If $q$ is a closed embedding, then $q$ induces an isomorphism $G_{1,2} \stackrel{\sim}{\rightarrow} G_{0}$, and therefore $G_{0}$ is a reductive, closed subgroup scheme of $G$. Thus to finish the proof of (b), we only have to show that the homomorphism $q$ is a closed embedding.

To check that $q$ is a closed embedding, it suffices to check that the fibers of $q$ are closed embeddings (see Theorem 2.5). For this we can assume that $A$ is a complete discrete valuation ring which has an algebraically closed residue field $k$; this implies that $G_{0}$ is a flat, closed subgroup scheme of $G$. Let $\mathfrak{n}:=\operatorname{Lie}\left(\operatorname{Ker}\left(q_{k}\right)\right)$. From Proposition 2.5.2(a) and Lemma 2.4 we get that either (iii) $\mathfrak{n}=0$ or (iv) $\operatorname{char}(k)=2$ and there exists a normal subgroup $F_{k}$ of $G_{1,2, k}$ which is isomorphic to $\mathrm{SO}_{2 n+1, k}$ for some $n \in \mathbb{N}^{*}$ and for which we have $\operatorname{Lie}\left(F_{k}\right) \cap \mathfrak{n} \neq 0$. We show that the assumption that condition (iv) holds leads to a contradiction. Let $F$ be a normal, closed subgroup scheme of $G_{1,2}$ that lifts $F_{k}$ and that is isomorphic to $\mathrm{SO}_{2 n+1, A}$ (see the last paragraph of the proof of Proposition 2.5.2(c)). Let $j_{0} \in\{1,2\}$ be such that $F \triangleleft G_{j_{0}} \triangleleft G_{1,2}$ (if condition (ii) holds, then $j_{0}=2$ ). As $G_{j_{0}}$ is a closed subgroup scheme of $G$, we have $\operatorname{Lie}\left(G_{j_{0}, k}\right) \cap \mathfrak{n}=0$ and therefore also $\operatorname{Lie}\left(F_{k}\right) \cap \mathfrak{n}=0$. Contradiction. Thus condition (iv) does not hold, and therefore condition (iii) holds. Consequently, $\operatorname{Ker}\left(q_{k}\right)$ has a trivial Lie algebra, and so it is a finite, étale, normal subgroup of $G_{1,2, k}$. Thus $\operatorname{Ker}\left(q_{k}\right)$ is a subgroup of $Z\left(G_{1,2, k}\right)$ and therefore also of each maximal torus of $G_{1,2, k}$. From this and Proposition 2.5.2(a) we get that $\operatorname{Ker}\left(q_{k}\right)$ is trivial. Therefore, $q_{k}$ is a closed embedding; thus $q$ is a closed embedding. 


\section{Acknowledgements}

We would like to thank the University of Utah, University of Arizona, Binghamton University, and the Institut des Hautes Études Scientifiques for good working conditions. We thank J.-P. Serre and A. Langer for pointing out the reference [Colliot-Thélène and Sansuc 1979] and O. Gabber for an email that independently outlined parts of the same paper; these led to a shorter paper and to stronger forms of Theorems 1.2 and 1.4 and of Proposition 5.1, for those reductive group schemes whose adjoints have geometric fibers that have simple factors of exceptional Lie types. We would like to thank J.-L. Colliot-Thélène for some comments and J. E. Humphreys for pointing out the references quoted in Remark 3.6(a). We would like to thank O. Gabber also for many other valuable comments and suggestions; Section 3 is mainly based on these suggestions. This research was partially supported by NSF grant DMS \#0900967.

\section{References}

[Block and Zassenhaus 1964] R. E. Block and H. Zassenhaus, "The Lie algebras with a nondegenerate trace form”, Illinois J. Math. 8 (1964), 543-549. MR 29 \#4776 Zbl 0131.27102

[Borel 1991] A. Borel, Linear algebraic groups, 2nd ed., Graduate Texts in Mathematics 126, Springer, New York, 1991. MR 92d:20001 Zbl 0726.20030

[Bosch et al. 1990] S. Bosch, W. Lütkebohmert, and M. Raynaud, Néron models, Ergebnisse der Mathematik (3) 21, Springer, Berlin, 1990. MR 91i:14034 Zbl 0705.14001

[Bourbaki 1989] N. Bourbaki, Lie groups and Lie algebras, Chapters 1-3, Springer, Berlin, 1989. Translated from the 1975 French 2nd edition. MR 89k:17001 Zbl 0672.22001

[Bourbaki 2002] N. Bourbaki, Lie groups and Lie algebras, Chapters 4-6, Springer, Berlin, 2002. Translated from the 1968 French original. MR 2003a:17001 Zbl 0983.17001

[Brown 1969] G. Brown, "Lie algebras of characteristic three with nondegenerate Killing form", Trans. Amer. Math. Soc. 137 (1969), 259-268. MR 39\#2825 Zbl 0176.30902

[Cartan and Eilenberg 1956] H. Cartan and S. Eilenberg, Homological algebra, Princeton University Press, 1956. MR 17,1040e Zbl 0075.24305

[Colliot-Thélène and Sansuc 1979] J.-L. Colliot-Thélène and J.-J. Sansuc, "Fibrés quadratiques et composantes connexes réelles", Math. Ann. 244:2 (1979), 105-134. MR 81c:14010 Zbl 0418.14016

[Curtis 1957] C. W. Curtis, "Modular Lie algebras, II”, Trans. Amer. Math. Soc. 86 (1957), 91-108. MR 20 \#933 Zbl 0078.02302

[Faltings 1999] G. Faltings, "Integral crystalline cohomology over very ramified valuation rings", $J$. Amer. Math. Soc. 12:1 (1999), 117-144. MR 99e:14022 Zbl 0914.14009

[Faltings and Chai 1990] G. Faltings and C.-L. Chai, Degeneration of abelian varieties, Ergebnisse der Mathematik (3) 22, Springer, Berlin, 1990. MR 92d:14036 Zbl 0744.14031

[Grothendieck 1966] A. Grothendieck, "Éléments de géométrie algébrique, IV: Étude locale des schémas et des morphismes de schémas III", Inst. Hautes Études Sci. Publ. Math. 28 (1966), 255. MR 36 \#178 Zbl 0144.19904 
[Grothendieck 1967] A. Grothendieck, "Éléments de géométrie algébrique, IV: Étude locale des schémas et des morphismes de schémas IV”, Inst. Hautes Études Sci. Publ. Math. 32 (1967), 361. MR 39 \#220 Zbl 0153.22301

[Helgason 1978] S. Helgason, Differential geometry, Lie groups, and symmetric spaces, Pure and Applied Mathematics 80, Academic Press, New York-London, 1978. MR 80k:53081 Zbl 0451.53038

[Humphreys 1972] J. E. Humphreys, Introduction to Lie algebras and representation theory, Graduate Texts in Mathematics 9, Springer, New York-Berlin, 1972. MR 48 \#2197 Zbl 0254.17004

[Humphreys 1995] J. E. Humphreys, Conjugacy classes in semisimple algebraic groups, Mathematical Surveys and Monographs 43, American Mathematical Society, Providence, RI, 1995. MR 97i:20057 Zbl 0834.20048

[Jantzen 2003] J. C. Jantzen, Representations of algebraic groups, 2nd ed., Mathematical Surveys and Monographs 107, American Mathematical Society, Providence, RI, 2003. MR 2004h:20061 Zbl 1034.20041

[de Jong 1998] A. J. de Jong, "Homomorphisms of Barsotti-Tate groups and crystals in positive characteristic", Invent. Math. 134:2 (1998), 301-333. MR 2000f:14070a Zbl 0929.14029

[Matsumura 1980] H. Matsumura, Commutative algebra, 2nd ed., Mathematics Lecture Note Series 56, Benjamin/Cummings Publishing Co., Reading, MA, 1980. MR 82i:13003 Zbl 0441.13001

[Mills 1957] W. H. Mills, "Classical type Lie algebras of characteristic 5 and 7”, J. Math. Mech. 6 (1957), 559-566. MR 19,632a Zbl 0079.04902

[Mills and Seligman 1957] W. H. Mills and G. B. Seligman, "Lie algebras of classical type", J. Math. Mech. 6 (1957), 519-548. MR 19,631d Zbl 0079.04804

[Moret-Bailly 1985] L. Moret-Bailly, "Un théorème de pureté pour les familles de courbes lisses", C. R. Acad. Sci. Paris Sér. I Math. 300:14 (1985), 489-492. MR 86f:14006 Zbl 0591.14017

[Prasad and Yu 2006] G. Prasad and J.-K. Yu, "On quasi-reductive group schemes”, J. Algebraic Geom. 15:3 (2006), 507-549. MR 2007c:14047 Zbl 1112.14053

[Seligman 1967] G. B. Seligman, Modular Lie algebras, Springer, New York, 1967. MR 39 \#6933 Zbl 0189.03201

[SGA 2 1968] A. Grothendieck, Cohomologie locale des faisceaux cohérents et théorèmes de Lefschetz locaux et globaux (Séminaire de Géométrie Algébrique du Bois Marie 1962), Advanced Stud. in Pure Math. 2, North-Holland, Amsterdam, 1968. MR 57 \#16294 Zbl 0197.47202

[SGA $3_{\text {I }}$ 1970] M. Demazure and A. Grothendieck, Schémas en groupes, Tome I: Propriétés générales des schémas en groupes, Exposés I-VII (Séminaire de Géométrie Algébrique du Bois Marie 19621964), Lecture Notes in Math. 151, Springer, Berlin, 1970. MR 43 \#223a Zbl 0207.51401

[SGA 3 II 1970] M. Demazure and A. Grothendieck, Schémas en groupes, Tome II: Groupes de type multiplicatif, et structure des schémas en groupes généraux, Exposés VIII-XVIII (Séminaire de Géométrie Algébrique du Bois Marie 1962-1964), Lecture Notes in Math. 152, Springer, Berlin, 1970. MR 43 \#223b Zbl 0209.24201

[SGA 3 III 1970] M. Demazure and A. Grothendieck, Schémas en groupes, Tome III: Structure des schémas en groupes réductifs, Exposés XIX-XXVI (Séminaire de Géométrie Algébrique du Bois Marie 1962-1964), Lecture Notes in Math. 153, Springer, Berlin, 1970. MR 43 \#223c Zbl 0212.52810

[Vasiu 1999] A. Vasiu, "Integral canonical models of Shimura varieties of preabelian type", Asian J. Math. 3:2 (1999), 401-518. MR 2002b:11087 Zbl 1002.11052

[Vasiu 2004] A. Vasiu, "A purity theorem for abelian schemes", Michigan Math. J. 52:1 (2004), 71-81. MR 2005f:14089 Zbl 1069.14049 
[Vasiu 2005a] A. Vasiu, "Normal, unipotent subgroup schemes of reductive groups", C. R. Math. Acad. Sci. Paris 341:2 (2005), 79-84. MR 2006g:14076 Zbl 1128.14033

[Vasiu 2005b] A. Vasiu, "On two theorems for flat, affine group schemes over a discrete valuation ring”, Cent. Eur. J. Math. 3:1 (2005), 14-25. MR 2005k:14097 Zbl 1108.14034

[Vasiu 2008] A. Vasiu, "Three methods to prove the existence of integral canonical models of Shimura varieties of Hodge type", preprint, 2008. arXiv 0811.2970

[Vasiu 2012a] A. Vasiu, "Good reductions of Shimura varieties of Hodge type in arbitrary unramified mixed characteristic, I”, preprint, 2012. arXiv 0707.1668

[Vasiu 2012b] A. Vasiu, "Good reductions of Shimura varieties of Hodge type in arbitrary unramified mixed characteristic, II”, preprint, 2012. arXiv 0712.1572

[Vasiu 2012c] A. Vasiu, "Integral models in unramified mixed characteristic $(0,2)$ of Hermitian orthogonal Shimura varieties of PEL type, I", J. Ramanujan Math. Soc. 27:4 (2012), 425-477. MR 3027446 Zbl 06187620

[Vasiu and Zink 2010] A. Vasiu and T. Zink, "Purity results for $p$-divisible groups and abelian schemes over regular bases of mixed characteristic", Doc. Math. 15 (2010), 571-599. MR 2012g:11113 Zbl 1252.11050

Communicated by Brian Conrad

Received 2015-01-06 Revised 2015-12-11 Accepted 2015-12-15

adrian@math.binghamton.edu Department of Mathematical Sciences, Binghamton University, P.O. Box 6000, Binghamton, NY 13902-6000, United States 


\section{Algebra \& Number Theory}

msp.org/ant

\section{EDITORS}

MANAGING EDITOR

Bjorn Poonen

Massachusetts Institute of Technology

Cambridge, USA

\author{
EDITORIAL BOARD CHAIR \\ David Eisenbud \\ University of California \\ Berkeley, USA
}

BOARD OF EDITORS

Georgia Benkart

Dave Benson

Richard E. Borcherds

John H. Coates

J-L. Colliot-Thélène

Brian D. Conrad

Hélène Esnault

Hubert Flenner

Sergey Fomin

Edward Frenkel

Andrew Granville

Joseph Gubeladze

Roger Heath-Brown

Craig Huneke

Kiran S. Kedlaya

János Kollár

Yuri Manin

Philippe Michel
University of Wisconsin, Madison, USA

University of Aberdeen, Scotland

University of California, Berkeley, USA

University of Cambridge, UK

CNRS, Université Paris-Sud, France

Stanford University, USA

Freie Universität Berlin, Germany

Ruhr-Universität, Germany

University of Michigan, USA

University of California, Berkeley, USA

Université de Montréal, Canada

San Francisco State University, USA

Oxford University, UK

University of Virginia, USA

Univ. of California, San Diego, USA

Princeton University, USA

Northwestern University, USA

École Polytechnique Fédérale de Lausanne
Susan Montgomery

Shigefumi Mori

Raman Parimala

Jonathan Pila

Anand Pillay

Victor Reiner

Peter Sarnak

Joseph H. Silverman

Michael Singer

Vasudevan Srinivas

J. Toby Stafford

Ravi Vakil

Michel van den Bergh

Marie-France Vignéras

Kei-Ichi Watanabe

Efim Zelmanov

Shou-Wu Zhang
University of Southern California, USA

RIMS, Kyoto University, Japan

Emory University, USA

University of Oxford, UK

University of Notre Dame, USA

University of Minnesota, USA

Princeton University, USA

Brown University, USA

North Carolina State University, USA

Tata Inst. of Fund. Research, India

University of Michigan, USA

Stanford University, USA

Hasselt University, Belgium

Université Paris VII, France

Nihon University, Japan

University of California, San Diego, USA

Princeton University, USA

\section{PRODUCTION}

production@msp.org

Silvio Levy, Scientific Editor

See inside back cover or msp.org/ant for submission instructions.

The subscription price for 2016 is US $\$ 290 /$ year for the electronic version, and $\$ 485 /$ year ( $+\$ 55$, if shipping outside the US) for print and electronic. Subscriptions, requests for back issues and changes of subscribers address should be sent to MSP.

Algebra \& Number Theory (ISSN 1944-7833 electronic, 1937-0652 printed) at Mathematical Sciences Publishers, 798 Evans Hall \#3840, c/o University of California, Berkeley, CA 94720-3840 is published continuously online. Periodical rate postage paid at Berkeley, CA 94704, and additional mailing offices.

ANT peer review and production are managed by EditFLOW ${ }^{\circledR}$ from MSP.

\section{PUBLISHED BY}

- mathematical sciences publishers

nonprofit scientific publishing

http://msp.org/

(C) 2016 Mathematical Sciences Publishers 


\section{Algebra \& Number Theory}

Volume $10 \quad$ No. $1 \quad 2016$

Stable sets of primes in number fields

ALEXANDER IVANOV

Hopf-Galois structures arising from groups with unique subgroup of order $p$

Tiмотну KонL

On tensor factorizations of Hopf algebras

Marc Keilberg and Peter Schauenburg

Extension theorems for reductive group schemes

ADRIAN VASIU

Actions of some pointed Hopf algebras on path algebras of quivers

RYAN Kinser and CHELSEA WALTON

On the image of the Galois representation associated to a non-CM Hida family

JACLYN LANG

Linear relations in families of powers of elliptic curves

FABRIZIO BARROERO and LAURA CAPUANO 